\title{
Rhodium-Catalyzed Asymmetric 1,6-Addition of Aryltitanates to Enynones Giving Axially Chiral Allenes
}

Tamio Hayashi,* Norihito Tokunaga, and Kazuya Inoue

Department of Chemistry, Graduate School of Science, Kyoto University, Sakyo, Kyoto 6068502, Japan

\section{Supporting Data}

General. All anaerobic and moisture-sensitive manipulations were carried out with standard Schlenk techniques under predried nitrogen or glovebox techniques under prepurified argon. NMR spectra were recorded on a JEOL JNM LA-500 spectrometer $\left(500 \mathrm{MHz}\right.$ for ${ }^{1} \mathrm{H}$, and $125 \mathrm{MHz}$ for ${ }^{13} \mathrm{C}$ ). Chemical shifts are reported in $\delta \mathrm{ppm}$ referenced to an internal $\mathrm{SiMe}_{4}$ standard for ${ }^{1} \mathrm{H}$ NMR and chloroform-d $\left(\delta\right.$ 77.00) for ${ }^{13} \mathrm{C}$ NMR. Optical rotations were measured on a JASCO DIP-370 polarimeter. The high-resolution mass spectra (HRMS) were performed on Applied Biosystems Mariner 8295 API-TOF workstation (ESI-TOF) and JEOL JMS-HX110 spectrometer (FAB).

Materials. THF and $\mathrm{Et}_{2} \mathrm{O}$ were distilled from benzophenone-ketyl under nitrogen prior to use. $\left.\left[\mathrm{RhCl}\left(\mathrm{C}_{2} \mathrm{H}_{4}\right)_{2}\right)\right]_{2},{ }^{1} \mathrm{~N}$-(2-pyridyl)triflimide, ${ }^{2}$ phenyltitanium triisopropoxide $(\mathrm{PhTi}(\mathrm{OPr}-$ i) $\left.{ }_{3}\right),{ }^{3} p$-methoxyphenyltitanium triisopropoxide $\left(4-\mathrm{MeOC}_{6} \mathrm{H}_{4} \mathrm{Ti}(\mathrm{OPr}-i)_{3}\right),{ }^{3} p-$ fluorophenyltitanium triisopropoxide $\left(4-\mathrm{FC}_{6} \mathrm{H}_{4} \mathrm{Ti}(\mathrm{OPr}-i){ }_{3}\right),{ }^{4}$ and lithium isopropoxide ${ }^{4}$ were prepared according to the reported procedures. $\mathrm{Ti}(\mathrm{OPr}-i)_{4}$, chlorotrimethylsilane, and pivaloyl chloride were distilled before use. Phenyllithium in cyclohexane/Et $2 \mathrm{O}, n$-butyllithium in hexane, methyllithium in $\mathrm{Et}_{2} \mathrm{O}$, bromobenzene, 4-bromoanisole, and 1-bromo-4-fluorobenzene were purchased.

Preparation of Enynones 1a-1d. Enynones 1a-1d were prepared from 3-( $p$ toluenesulfonyl)-2-cyclohexen-1-one according to the reported procedures. 5 - $(\boldsymbol{p}$ Toluenesulfonyl)-2-cyclohexen-1-one: This compound was prepared according to the reported procedures, ${ }^{5}$ and used without further purification. ${ }^{1} \mathrm{H} \mathrm{NMR}\left(\mathrm{CDCl}_{3}\right) \delta 1.95$ (quintet, 
$J=6.5 \mathrm{~Hz}, 2 \mathrm{H}), 2.29(\mathrm{t}, J=6.5 \mathrm{~Hz}, 2 \mathrm{H}), 2.42(\mathrm{~s}, 3 \mathrm{H}), 2.45(\mathrm{td}, J=6.3,1.1 \mathrm{~Hz}, 2 \mathrm{H}), 5.76$ $(\mathrm{t}, J=1.1 \mathrm{~Hz}, 1 \mathrm{H}), 7.34(\mathrm{~d}, J=8.5 \mathrm{~Hz}, 2 \mathrm{H}), 7.78(\mathrm{~d}, J=8.5 \mathrm{~Hz}, 2 \mathrm{H}) .{ }^{13} \mathrm{C} \mathrm{NMR}\left(\mathrm{CDCl}_{3}\right) \delta$ $20.74,21.67,28.55,36.35,116.74,128.15,130.07,132.44,146.12,168.15,198.57$. HRMS (ESI-TOF) Calcd for $\mathrm{C}_{13} \mathrm{H}_{14} \mathrm{O}_{4} \mathrm{SNa}: 289.0510\left([\mathrm{M}+\mathrm{Na}]^{+}\right)$. Found: 289.0506 $\left([\mathrm{M}+\mathrm{Na}]^{+}\right)$. 3-(1-Hexynyl)-2-cyclohexen-1-one (1a): purified by silica gel chromatography $($ Hexane/AcOEt $=7 / 1)$ and bulb-to-bulb distillation $(87 \%$ yield $) ;{ }^{1} \mathrm{H}$ NMR $\left(\mathrm{CDCl}_{3}\right) \delta 0.93(\mathrm{t}, J=7.4 \mathrm{~Hz}, 3 \mathrm{H}), 1.39-1.48(\mathrm{~m}, 2 \mathrm{H}), 1.52-1.59(\mathrm{~m}, 2 \mathrm{H}), 2.01$ (quintet, $J=$ $6.2 \mathrm{~Hz}, 2 \mathrm{H}), 2.39(\mathrm{t}, J=7.1 \mathrm{~Hz}, 2 \mathrm{H}), 2.40(\mathrm{t}, J=6.8 \mathrm{~Hz}, 2 \mathrm{H}), 2.41(\mathrm{td}, J=6.2,1.7 \mathrm{~Hz}$, $2 \mathrm{H}), 6.13(\mathrm{t}, J=1.7 \mathrm{~Hz}, 1 \mathrm{H}) .{ }^{13} \mathrm{C} \mathrm{NMR}\left(\mathrm{CDCl}_{3}\right) \delta 13.45,19.40,21.87,22.50,30.27$, 30.79, 37.20, 80.35, 102.27, 131.76, 144.51, 198.83. HRMS (ESI-TOF) Calcd for $\mathrm{C}_{12} \mathrm{H}_{17} \mathrm{O}: 177.1279\left([\mathrm{M}+\mathrm{H}]^{+}\right)$. Found: $177.1281\left([\mathrm{M}+\mathrm{H}]^{+}\right)$. 3-(Cyclohexylethynyl)-2cyclohexen-1-one (1b): purified by silica gel chromatography (Hexane/AcOEt $=4 / 1)$ and bulb-to-bulb distillation (55\% yield); ${ }^{1} \mathrm{H}$ NMR $\left(\mathrm{CDCl}_{3}\right) \delta$ 1.29-1.39 (m, 3H), 1.44-1.57 (m, $3 \mathrm{H}), 1.67-1.75(\mathrm{~m}, 2 \mathrm{H}), 1.79-1.87(\mathrm{~m}, 2 \mathrm{H}), 2.01$ (quintet, $J=6.1 \mathrm{~Hz}, 2 \mathrm{H}), 2.39(\mathrm{t}, J=6.1$ $\mathrm{Hz}, 2 \mathrm{H}), 2.42(\mathrm{td}, J=6.1,1.6 \mathrm{~Hz}, 2 \mathrm{H}), 2.58(\mathrm{tt}, J=9.0,3.9 \mathrm{~Hz}, 1 \mathrm{H}), 6.14(\mathrm{t}, J=1.6 \mathrm{~Hz}$, 1H). ${ }^{13} \mathrm{C} \mathrm{NMR}\left(\mathrm{CDCl}_{3}\right) \delta 22.55,24.69,25.71,29.94,30.93,32.16,37.24,80.40,106.14$, 131.75, 144.58, 198.84. HRMS (ESI-TOF) Calcd for $\mathrm{C}_{14} \mathrm{H}_{19} \mathrm{O}: 203.1435\left([\mathrm{M}+\mathrm{H}]^{+}\right)$. Found: $203.1425\left([\mathrm{M}+\mathrm{H}]^{+}\right)$. 3-(tert-Butylethynyl)-2-cyclohexen-1-one (1c): purified by silica gel chromatography (Hexane/AcOEt = 6/1) and bulb-to-bulb distillation ( $86 \%$ yield); This product was characterized by comparison of the spectroscopic data with those reported previously. 6 3-(4-Methoxyphenylethynyl)-2-cyclohexen-1-one (1d): purified by silica gel chromatography $(\mathrm{Hexane} / \mathrm{AcOEt}=4 / 1)$ and bulb-to-bulb distillation $(52 \%$ yield $) ;{ }^{1} \mathrm{H}$ $\mathrm{NMR}\left(\mathrm{CDCl}_{3}\right) \delta 2.07$ (quintet, $\left.J=6.5 \mathrm{~Hz}, 2 \mathrm{H}\right), 2.44(\mathrm{t}, J=6.5 \mathrm{~Hz}, 2 \mathrm{H}), 2.54(\mathrm{td}, J=6.5$, $1.6 \mathrm{~Hz}, 2 \mathrm{H}), 3.83(\mathrm{~s}, 3 \mathrm{H}), 6.26(\mathrm{t}, J=1.6 \mathrm{~Hz}, 1 \mathrm{H}), 6.88(\mathrm{~d}, J=8.9 \mathrm{~Hz}, 2 \mathrm{H}), 7.43(\mathrm{~d}, J=$ $8.9 \mathrm{~Hz}, 2 \mathrm{H}) .{ }^{13} \mathrm{C} \mathrm{NMR}\left(\mathrm{CDCl}_{3}\right) \delta 22.64,30.60,37.32,55.34,87.67,100.35,114.04$, 114.19, 131.69, 133.64, 143.77, 160.61, 198.68. HRMS (ESI-TOF) Calcd for $\mathrm{C}_{15} \mathrm{H}_{15} \mathrm{O}_{2}$ : $227.1071\left([\mathrm{M}+\mathrm{H}]^{+}\right)$. Found: $227.1066\left([\mathrm{M}+\mathrm{H}]^{+}\right)$. 
Preparation of Enynone 1e. 3-(p-Toluenesulfonyl)-2-cyclopenten-1-one was prepared from 1,3-cyclopentanedione $(1.82 \mathrm{~g}, 18.6 \mathrm{mmol})$ in a manner similar to 3 - $(p$ toluenesulfonyl)-2-cyclohexen-1-one. ${ }^{5}$ To a slurry of $\operatorname{LiBr}(2.42 \mathrm{~g}, 27.9 \mathrm{mmol}, 1.5 \mathrm{eq})$, $\mathrm{Pd}\left(\mathrm{PPh}_{3}\right)_{4}(1.03 \mathrm{~g}, 0.891 \mathrm{mmol}, 4.8 \mathrm{~mol} \% \mathrm{Pd})$, and 3-( $p$-toluenesulfonyl)-2-cyclopenten-1one in THF (30 mL) was added a solution of 1-hexynyltributyltin $(9.39 \mathrm{~g}, 25.2 \mathrm{mmol})$ in THF (30 mL). The resulting mixture was heated to $80{ }^{\circ} \mathrm{C}$ for $48 \mathrm{~h}$ and cooled to room temperature. After concentrated in vacuo, the mixture was diluted with $\mathrm{CH}_{2} \mathrm{Cl}_{2}$ and washed with water. The aqueous layer was extracted twice with $\mathrm{CH}_{2} \mathrm{Cl}_{2}$, and the combined organic layers were washed with a $10 \% \mathrm{NH}_{3}$ solution, water, and saturated $\mathrm{NaCl}$, before it was dried over $\mathrm{MgSO}_{4}$ and filtered through a short pad of silica gel. After removal of the solvent, the crude mixture was purified by silica gel chromatography (Hexane/AcOEt $=3 / 1)$, followed by bulb-to-bulb distillation to give enynone $\mathbf{1 e}$ as colorless oil (1.60 g, 53\% yield).; $\mathbf{3 - ( p -}$ Toluenesulfonyl)-2-cyclopenten-1-one: ${ }^{1} \mathrm{H}$ NMR $\left(\mathrm{CDCl}_{3}\right) \delta 2.42-2.46(\mathrm{~m}, 2 \mathrm{H}), 2.48$ (s, 3H), 2.65-2.70 (m, 2H), $5.92(\mathrm{t}, J=1.5 \mathrm{~Hz}, 1 \mathrm{H}), 7.41$ (d, $J=8.5 \mathrm{~Hz}, 2 \mathrm{H}), 7.86(\mathrm{~d}, J=$ $8.5 \mathrm{~Hz}, 2 \mathrm{H}) .{ }^{13} \mathrm{C} \mathrm{NMR}\left(\mathrm{CDCl}_{3}\right) \delta 21.75,28.63,34.03,115.02,128.40,130.25,131.69$, 146.69, 178.91, 204.71. HRMS (ESI-TOF) Calcd for $\mathrm{C}_{12} \mathrm{H}_{12} \mathrm{O}_{4} \mathrm{SNa}: 275.0353\left([\mathrm{M}+\mathrm{Na}]^{+}\right)$. Found: $275.0339\left([\mathrm{M}+\mathrm{Na}]^{+}\right)$. 3-(1-Hexynyl)-2-cyclopenten-1-one (1e): ${ }^{1} \mathrm{H}$ NMR $\left(\mathrm{CDCl}_{3}\right) \delta 0.94(\mathrm{t}, J=7.4 \mathrm{~Hz}, 3 \mathrm{H}), 1.40-1.49(\mathrm{~m}, 2 \mathrm{H}), 1.55-1.62(\mathrm{~m}, 2 \mathrm{H}), 2.39-2.43(\mathrm{~m}$, $2 \mathrm{H}), 2.46(\mathrm{t}, J=7.1 \mathrm{~Hz}, 2 \mathrm{H}), 2.68-2.72(\mathrm{~m}, 2 \mathrm{H}), 6.19(\mathrm{t}, J=1.8 \mathrm{~Hz}, 1 \mathrm{H}) .{ }^{13} \mathrm{C}$ NMR $\left(\mathrm{CDCl}_{3}\right) \delta 13.42,19.58,21.88,30.14,32.78,34.66,76.92,107.56,135.20,158.32$, 209.64. HRMS (ESI-TOF) Calcd for $\mathrm{C}_{11} \mathrm{H}_{15} \mathrm{O}: 163.1122\left([\mathrm{M}+\mathrm{H}]^{+}\right)$. Found: 163.1127 $\left([\mathrm{M}+\mathrm{H}]^{+}\right)$.

\section{Rhodium-Catalyzed Asymmetric 1,6-Addition of Aryltitanates (ArTi(OPr-} i) ${ }_{4} \mathrm{Li}$, (2)) to Enynones 1 in the Presence of Chlorotrimethylsilane. Aryltitanate $\operatorname{ArTi}(\mathrm{OPr}-i)_{4} \mathrm{Li}(\mathbf{2})$ was generated by either of the following three ways: (i) $\mathrm{Ti}(\mathrm{OPr}-i)_{4}(0.14$ $\mathrm{mL}, 0.48 \mathrm{mmol})$ was added to a solution of phenyllithium $(0.48 \mathrm{~mL}, 0.94 \mathrm{M}$ in cyclohexane/Et $2 \mathrm{O}, 0.45 \mathrm{mmol})$ in $\mathrm{THF}(0.30 \mathrm{~mL})$ at room temperature and the mixture was stirred at room temperature for $0.5 \mathrm{~h}$. (ii) $n$-Butyllithium $(0.29 \mathrm{~mL}, 1.56 \mathrm{M}$ in hexane, 0.45 
mmol $)$ was added to a solution of aryl bromide $\mathrm{ArBr}(0.45 \mathrm{mmol})$ in $^{\mathrm{Et}_{2} \mathrm{O}}(1.0 \mathrm{~mL})$ at $-78^{\circ} \mathrm{C}$ and the mixture was stirred at $0{ }^{\circ} \mathrm{C}$ for $1 \mathrm{~h}$. $\mathrm{Ti}(\mathrm{OPr}-i)_{4}(0.14 \mathrm{~mL}, 0.48 \mathrm{mmol})$ was added to the ArLi solution at room temperature and the mixture was stirred at room temperature for $0.5 \mathrm{~h}$. (iii) THF $(0.30 \mathrm{~mL})$ was added to ArTi(OPr- $i)_{3}(0.45 \mathrm{mmol})$ and LiOPr- $i(30 \mathrm{mg}, 0.45 \mathrm{mmol})$ and the solution was stirred at room temperature for $0.5 \mathrm{~h}$. To a solution of $\left.\left[\operatorname{RhCl}\left(\mathrm{C}_{2} \mathrm{H}_{4}\right)_{2}\right)\right]_{2}$ (5.8 mg, $0.15 \mathrm{mmol}, 10 \mathrm{~mol} \% \mathrm{Rh}$ ), $(R)$-segphos (20 mg, $0.33 \mathrm{mmol}, 1.1$ eq to $\mathrm{Rh}$ ), enynone $1(0.30 \mathrm{mmol})$, and chlorotrimethylsilane $(80 \mu \mathrm{L}, 0.63 \mathrm{mmol})$ in THF $(0.70 \mathrm{~mL})$ was added a solution of $\operatorname{ArTi}(\mathrm{OPr}-i)_{4} \mathrm{Li}(2)(0.45 \mathrm{mmol})$ at $20{ }^{\circ} \mathrm{C}$. After $0.5 \mathrm{~h}$ stirring at the same temperature, the mixture was concentrated under reduced pressure, treated with $\mathrm{Et}_{2} \mathrm{O}$ (ca. $3 \mathrm{~mL}$ ) and $\mathrm{H}_{2} \mathrm{O}$ (ca. $50 \mu \mathrm{L}$ ), and filtered through a short Celite/ $\mathrm{MgSO}_{4}$ pad (eluent: $\mathrm{Et}_{2} \mathrm{O}$ ). The filtrate was concentrated in vacuo to give the crude allenylalkenyl silyl ether 3 . The yield of the silyl ether 3 was determined by ${ }^{1} \mathrm{H}$ NMR analysis of the crude product. The spectroscopic data of the silyl enol ethers are shown below: 3-(2-Phenylhexenylidene)-1 (trimethylsilyloxy)cyclohexene (3am): ${ }^{1} \mathrm{H}$ NMR $\left(\mathrm{CDCl}_{3}\right) \delta 0.22(\mathrm{~s}, 9 \mathrm{H}), 0.92(\mathrm{t}, J=$ $7.5 \mathrm{~Hz}, 3 \mathrm{H}$ ), 1.40 (sextet, $J=7.5 \mathrm{~Hz}, 2 \mathrm{H}$ ), 1.49 (quintet, $J=7.4 \mathrm{~Hz}, 2 \mathrm{H}$ ), 1.79-1.91 (m, 2H), $2.16(\mathrm{t}, J=6.2 \mathrm{~Hz}, 2 \mathrm{H}), 2.32(\mathrm{ddd}, J=14.2,6.6,5.3 \mathrm{~Hz}, 1 \mathrm{H}), 2.38(\mathrm{ddd}, J=14.2$, 8.8, $6.8 \mathrm{~Hz}, 1 \mathrm{H}), 2.42(\mathrm{t}, J=7.5 \mathrm{~Hz}, 2 \mathrm{H}), 5.34(\mathrm{~s}, 1 \mathrm{H}), 7.16(\mathrm{tt}, J=7.4,1.5 \mathrm{~Hz}, 1 \mathrm{H}), 7.29$ $(\mathrm{t}, J=7.4 \mathrm{~Hz}, 2 \mathrm{H}), 7.37(\mathrm{~d}, J=7.4 \mathrm{~Hz}, 2 \mathrm{H}) .{ }^{13} \mathrm{C} \mathrm{NMR}\left(\mathrm{CDCl}_{3}\right) \delta 0.37,14.07,22.40$, $22.94,27.17,30.04,30.08,103.68,104.20,105.56,126.09,126.13,128.18,138.24$, 152.76, 202.14. $\quad 3$ - ( 2 - ( 4 - F l u o r o p h e n y l ) h ex e n y lide n e ) - 1 (trimethylsilyloxy)cyclohexene (3an): ${ }^{1} \mathrm{H}$ NMR $\left(\mathrm{CDCl}_{3}\right) \delta 0.22(\mathrm{~s}, 9 \mathrm{H}), 0.91(\mathrm{t}, J=$ $7.3 \mathrm{~Hz}, 3 \mathrm{H}$ ), 1.39 (sextet, $J=7.3 \mathrm{~Hz}, 2 \mathrm{H}$ ), 1.47 (quintet, $J=7.4 \mathrm{~Hz}, 2 \mathrm{H}$ ), 1.85 (quintet, $J=$ $6.2 \mathrm{~Hz}, 2 \mathrm{H}), 2.16(\mathrm{t}, J=6.2 \mathrm{~Hz}, 2 \mathrm{H}), 2.29-2.39(\mathrm{~m}, 2 \mathrm{H}), 2.39(\mathrm{t}, J=7.3 \mathrm{~Hz}, 2 \mathrm{H}), 5.33(\mathrm{t}, J$ $=1.1 \mathrm{~Hz}, 1 \mathrm{H}), 6.97(\mathrm{t}, J=8.8 \mathrm{~Hz}, 2 \mathrm{H}), 7.31(\mathrm{dd}, J=8.8,5.4 \mathrm{~Hz}, 2 \mathrm{H}) .{ }^{13} \mathrm{C} \mathrm{NMR}\left(\mathrm{CDCl}_{3}\right)$ $\delta 0.33,14.01,22.35,22.92,27.19,29.97,30.08,30.29,103.58,104.46,104.78,114.93$ $(\mathrm{d}, J=21.6 \mathrm{~Hz}), 127.49(\mathrm{~d}, J=7.1 \mathrm{~Hz}), 134.20(\mathrm{~d}, J=3.1 \mathrm{~Hz}), 152.99,161.52(\mathrm{~d}, J=$ $244.1 \mathrm{~Hz}), 201.82(\mathrm{~d}, J=2.0 \mathrm{~Hz}) . \quad$ 3-(2-(4-Methoxyphenyl)hexenylidene)-1(trimethylsilyloxy)cyclohexene (3ao): ${ }^{1} \mathrm{H}$ NMR $\left(\mathrm{CDCl}_{3}\right) \delta 0.22(\mathrm{~s}, 9 \mathrm{H}), 0.91(\mathrm{t}, J=$ 
$7.2 \mathrm{~Hz}, 3 \mathrm{H}$ ), 1.39 (sextet, $J=7.2 \mathrm{~Hz}, 2 \mathrm{H}$ ), 1.48 (quintet, $J=7.3 \mathrm{~Hz}, 2 \mathrm{H}$ ), 1.84 (quintet, $J=$ $6.0 \mathrm{~Hz}, 2 \mathrm{H}), 2.15(\mathrm{t}, J=6.4 \mathrm{~Hz}, 2 \mathrm{H}), 2.28-2.39(\mathrm{~m}, 2 \mathrm{H}), 2.39(\mathrm{t}, J=7.2 \mathrm{~Hz}, 2 \mathrm{H}), 3.80(\mathrm{~s}$, $3 \mathrm{H}), 5.33(\mathrm{~s}, 1 \mathrm{H}), 6.84(\mathrm{~d}, J=8.9 \mathrm{~Hz}, 2 \mathrm{H}), 7.29(\mathrm{~d}, J=8.9 \mathrm{~Hz}, 2 \mathrm{H}) .{ }^{13} \mathrm{C} \mathrm{NMR}\left(\mathrm{CDCl}_{3}\right) \delta$ $0.33,14.05,22.38,22.94,27.29,30.02,30.07,30.24,55.21,103.99,104.02,105.01$, 113.64, 127.10, 152.56, 158.17, 201.43. 3-(2-Cyclohexyl-2-phenylethenylidene)-1(trimethylsilyloxy)cyclohexene $(\mathbf{3 b m}):{ }^{1} \mathrm{H}$ NMR $\left(\mathrm{CDCl}_{3}\right) \delta 0.22(\mathrm{~s}, 9 \mathrm{H}), 1.08-1.18$ $(\mathrm{m}, 2 \mathrm{H}), 1.18-1.23(\mathrm{~m}, 1 \mathrm{H}), 1.36(\mathrm{qt}, J=12.7,3.2 \mathrm{~Hz}, 2 \mathrm{H}), 1.65-1.72(\mathrm{~m}, 1 \mathrm{H}), 1.73-1.79$ (m, 2H), 1.79-1.91 (m, 4H), $2.15(\mathrm{td}, J=6.3,0.8 \mathrm{~Hz}, 2 \mathrm{H}), 2.32(\mathrm{ddd}, J=14.5,7.0,5.2$ $\mathrm{Hz}, 1 \mathrm{H}), 2.37(\mathrm{ddd}, J=14.5,7.2,5.4 \mathrm{~Hz}, 1 \mathrm{H}), 2.45(\mathrm{tt}, J=11.3,3.2 \mathrm{~Hz}, 1 \mathrm{H}), 5.34(\mathrm{t}, J=$ $0.8 \mathrm{~Hz}, 1 \mathrm{H}), 7.16(\mathrm{tt}, J=7.3,1.1 \mathrm{~Hz}, 1 \mathrm{H}), 7.29(\mathrm{t}, J=7.3 \mathrm{~Hz}, 2 \mathrm{H}), 7.35(\mathrm{~d}, J=7.3 \mathrm{~Hz}$, 2H). ${ }^{13} \mathrm{C} \mathrm{NMR}\left(\mathrm{CDCl}_{3}\right) \delta 0.39,22.97,26.43,26.65,27.36,30.08,33.13,38.66,104.06$, 104.88, 112.09, 126.08, 126.56, 128.19, 137.90, 152.55, 201.89. 3-(2-Cyclohexyl-2(4-methoxyphenyl)ethenylidene)-1-(trimethylsilyloxy)cyclohexene $\quad(3 \mathrm{bo}):{ }^{1} \mathrm{H}$ $\operatorname{NMR}\left(\mathrm{CDCl}_{3}\right) \delta 0.22(\mathrm{~s}, 9 \mathrm{H}), 1.07-1.28(\mathrm{~m}, 3 \mathrm{H}), 1.35(\mathrm{qt}, J=10.6,3.1 \mathrm{~Hz}, 2 \mathrm{H}), 1.63-1.80$ (m, 3H), 1.80-1.93 (m, 4H), $2.15(\mathrm{t}, J=6.3 \mathrm{~Hz}, 2 \mathrm{H}), 2.27-2.46(\mathrm{~m}, 2 \mathrm{H}), 2.40(\mathrm{tt}, J=11.2$, $3.2 \mathrm{~Hz}, 1 \mathrm{H}), 3.79(\mathrm{~s}, 3 \mathrm{H}), 5.33(\mathrm{~s}, 1 \mathrm{H}), 6.84(\mathrm{~d}, J=8.2 \mathrm{~Hz}, 2 \mathrm{H}), 7.27(\mathrm{~d}, J=8.2 \mathrm{~Hz}, 2 \mathrm{H})$. ${ }^{13} \mathrm{C}$ NMR $\left(\mathrm{CDCl}_{3}\right) \delta$ 0.42, 23.01, 26.47, 26.68, 27.51, 30.10, 33.13, 38.83, 55.30, 104.41, 104.76, 111.55, 113.72, 127.60, 129.40, 130.24, 152.40, 201.25. 3-(3,3-Dimethyl-2phenylbutenylidene)-1-(trimethylsilyloxy)cyclohexene $(3 \mathbf{c m}):{ }^{1} \mathrm{H} \mathrm{NMR}\left(\mathrm{CDCl}_{3}\right) \delta$ $0.21(\mathrm{~s}, 9 \mathrm{H}), 1.11$ (s, 9H), 1.77 (quintet, $J=6.3 \mathrm{~Hz}, 2 \mathrm{H}), 2.03-2.14(\mathrm{~m}, 2 \mathrm{H}), 2.23-2.34$ (m, $2 \mathrm{H}), 5.32(\mathrm{t}, J=1.1 \mathrm{~Hz}, 1 \mathrm{H}), 7.18-7.30(\mathrm{~m}, 5 \mathrm{H}) .{ }^{13} \mathrm{C} \mathrm{NMR}\left(\mathrm{CDCl}_{3}\right) \delta 0.39,22.74,27.63$, $29.99,30.19$, 35.24, 101.54, 105.05, 115.85, 126.12, 127.58, 129.45, 138.89, 151.97, 199.73. 3 - ( 2 - ( 4 - Me th ox y pheny 1$)$ - 2 - phen y let e n y lidene ) - 1 (trimethylsilyloxy)cyclohexene $(\mathbf{3 d m}):{ }^{1} \mathrm{H}$ NMR $\left(\mathrm{CDCl}_{3}\right) \delta 0.23(\mathrm{~s}, 9 \mathrm{H}), 1.86$ (quintet, $J=6.3 \mathrm{~Hz}, 2 \mathrm{H}), 2.16(\mathrm{td}, J=6.3,0.8 \mathrm{~Hz}, 2 \mathrm{H}), 2.44(\mathrm{t}, J=6.3 \mathrm{~Hz}, 2 \mathrm{H}), 3.81(\mathrm{~s}, 3 \mathrm{H}), 5.43$ $(\mathrm{t}, J=0.8 \mathrm{~Hz}, 1 \mathrm{H}), 6.86(\mathrm{~d}, J=8.8 \mathrm{~Hz}, 2 \mathrm{H}), 7.24(\mathrm{~d}, J=8.8 \mathrm{~Hz}, 2 \mathrm{H}), 7.28-7.35(\mathrm{~m}, 5 \mathrm{H})$. ${ }^{13} \mathrm{C} \mathrm{NMR}\left(\mathrm{CDCl}_{3}\right) \delta 0.15,22.50,27.00,29.82,54.98,102.88,103.81,109.15,113.41$, $126.45, \quad 127.89, \quad 128.21,129.36,129.99,137.99,153.32,203.94 . \quad 3-(2$ - 
Phenylhexenylidene)-1-(trimethylsilyloxy)cyclopentene (3em): ${ }^{1} \mathrm{H}$ NMR $\left(\mathrm{CDCl}_{3}\right)$ $\delta 0.26(\mathrm{~s}, 9 \mathrm{H}), 0.92(\mathrm{t}, J=7.5 \mathrm{~Hz}, 3 \mathrm{H}), 1.40$ (sextet, $J=7.5 \mathrm{~Hz}, 2 \mathrm{H}), 1.51$ (quintet, $J=7.5$ $\mathrm{Hz}, 2 \mathrm{H}), 2.43(\mathrm{t}, J=7.5 \mathrm{~Hz}, 2 \mathrm{H}), 2.46-2.54(\mathrm{~m}, 2 \mathrm{H}), 2.65-2.79(\mathrm{~m}, 2 \mathrm{H}), 5.16(\mathrm{t}, J=1.5$ $\mathrm{Hz}, 1 \mathrm{H}), 7.16(\mathrm{t}, J=7.5 \mathrm{~Hz}, 1 \mathrm{H}), 7.28(\mathrm{t}, J=7.5 \mathrm{~Hz}, 2 \mathrm{H}), 7.40(\mathrm{~d}, J=7.5 \mathrm{~Hz}, 2 \mathrm{H}) .{ }^{13} \mathrm{C}$ $\operatorname{NMR}\left(\mathrm{CDCl}_{3}\right) \delta-0.01,14.03,22.45,25.76,30.16,30.55,32.93,103.87,108.24,111.56$, $126.07,126.12,128.10,138.63,159.47,198.43$.

General Procedure for Conversion of Silyl Enol Ether 3 into Pivalate 4. To a solution of the crude silyl enol ether 3 in THF $(2.0 \mathrm{~mL})$ was added methyllithium $(0.49 \mathrm{~mL}, 0.92$ $\mathrm{M}$ in $\mathrm{Et}_{2} \mathrm{O}, 0.45 \mathrm{mmol}$ ) at $-78{ }^{\circ} \mathrm{C}$, and the mixture was stirred at $0{ }^{\circ} \mathrm{C}$ for $0.5 \mathrm{~h}$. To the solution, pivaloyl chloride $(74 \mu \mathrm{L}, 0.60 \mathrm{mmol})$ was added at $-78^{\circ} \mathrm{C}$, and the reaction mixture was stirred at room temperature for $0.5 \mathrm{~h}$. The solvent was removed in vacuo, and the residue was diluted with $\mathrm{Et}_{2} \mathrm{O}$. The solution was washed with saturated $\mathrm{NaHCO}_{3}$, before it was dried over $\mathrm{MgSO}_{4}$ and concentrated under reduced pressure. The crude product was purified by silica gel chromatography. The enantiomeric excess of the pivalate ester 4 was determined by HPLC analysis with a chiral stationary phase column (Daicel Chiralcel OD-H, eluent: Hexane). The results are summarized in Table 1. The NMR spectra, optical rotations , and the data of combustion analysis and HRMS of the pivalate esters are shown below: 3-(2-Phenylhexenylidene)-1(trimethylacetoxy)cyclohexene (4am): purified by PTLC (Hexane/AcOEt $=20 / 1)(85 \%$ yield); ${ }^{1} \mathrm{H} \mathrm{NMR}\left(\mathrm{CDCl}_{3}\right) \delta 0.92(\mathrm{t}, J=7.4 \mathrm{~Hz}, 3 \mathrm{H}), 1.25(\mathrm{~s}, 9 \mathrm{H}), 1.40$ (sextet, $\left.J=7.4 \mathrm{~Hz}, 2 \mathrm{H}\right)$, 1.46-1.54 (m, 2H), 1.85- $1.97(\mathrm{~m}, 2 \mathrm{H}), 2.30(\mathrm{td}, J=6.1,1.3 \mathrm{~Hz}, 2 \mathrm{H}), 2.37-2.49(\mathrm{~m}, 2 \mathrm{H}), 2.42$ (t, $J=7.4 \mathrm{~Hz}, 2 \mathrm{H}), 5.82(\mathrm{t}, J=1.3 \mathrm{~Hz}, 1 \mathrm{H}), 7.17(\mathrm{tt}, J=7.4,1.3 \mathrm{~Hz}, 1 \mathrm{H}), 7.28(\mathrm{t}, J=7.4 \mathrm{~Hz}$, 2H), $7.35(\mathrm{~d}, J=7.4 \mathrm{~Hz}, 2 \mathrm{H}) .{ }^{13} \mathrm{C} \mathrm{NMR}\left(\mathrm{CDCl}_{3}\right) \delta 13.97,22.43,22.58,26.85,27.03,27.08$, $29.95,30.03,38.83,102.53,106.45,112.96,126.15,126.48,128.23,137.32,149.50,176.83$, 203.65. $[\alpha]^{20} \mathrm{D}+19.2\left(c 1.05, \mathrm{CHCl}_{3}\right)$ for $92 \%$ ee. Anal. Calcd for $\mathrm{C}_{23} \mathrm{H}_{30} \mathrm{O}_{2}: \mathrm{C}, 81.61 ; \mathrm{H}$, 8.93. Found: C, $81.36 ; \quad H, 9.00 .3$ - ( 2 - ( 4 - Fluorophenyl)hexenylidene ) - 1 (trimethylacetoxy)cyclohexene (4an): purified by PTLC (Hexane/AcOEt = 15/1) (85\% yield); ${ }^{1} \mathrm{H} \mathrm{NMR}\left(\mathrm{CDCl}_{3}\right) \delta 0.92(\mathrm{t}, J=7.2 \mathrm{~Hz}, 3 \mathrm{H}), 1.25(\mathrm{~s}, 9 \mathrm{H}), 1.39$ (sextet, $J=7.2 \mathrm{~Hz}, 2 \mathrm{H}$ ), 1.43-1.53 (m, 2H), 1.92 (quintet, $J=6.3 \mathrm{~Hz}, 2 \mathrm{H}), 2.31(\mathrm{t}, J=6.3 \mathrm{~Hz}, 2 \mathrm{H}), 2.39(\mathrm{t}, J=7.2 \mathrm{~Hz}$, 
2H), 2.36-2.48 (m, 2H), $5.81(\mathrm{~s}, 1 \mathrm{H}), 6.97(\mathrm{t}, J=8.5 \mathrm{~Hz}, 2 \mathrm{H}), 7.30(\mathrm{dd}, J=8.5,5.4 \mathrm{~Hz}, 2 \mathrm{H})$. ${ }^{13} \mathrm{C} \mathrm{NMR}\left(\mathrm{CDCl}_{3}\right) \delta 13.95,22.40,22.55,26.84,27.01,27.08,29.94,30.15,38.84,102.76$, 105.61, 112.83, $115.04(\mathrm{~d}, J=21.6 \mathrm{~Hz}), 127.60(\mathrm{~d}, J=8.3 \mathrm{~Hz}), 133.27(\mathrm{~d}, J=3.1 \mathrm{~Hz})$, $149.71,161.66(\mathrm{~d}, J=244.1 \mathrm{~Hz}), 176.82,203.33(\mathrm{~d}, J=2.1 \mathrm{~Hz}) .[\alpha]^{20} \mathrm{D}+13.1(c 0.99$, $\mathrm{CHCl}_{3}$ ) for $91 \%$ ee. Anal. Calcd for $\mathrm{C}_{23} \mathrm{H}_{29} \mathrm{O}_{2} \mathrm{~F}: \mathrm{C}, 77.49 ; \mathrm{H}, 8.20$. Found: C, 77.27; H, 8.15. 3-(2-(4-Methoxyphenyl)hexenylidene)-1-(trimethylacetoxy)cyclohexene (4ao): purified by PTLC $(\mathrm{Hexane} / \mathrm{AcOEt}=7 / 1)(83 \%$ yield $) ;{ }^{1} \mathrm{H} \mathrm{NMR}\left(\mathrm{CDCl}_{3}\right) \delta 0.92(\mathrm{t}, J=7.3 \mathrm{~Hz}$, $3 \mathrm{H}), 1.25(\mathrm{~s}, 9 \mathrm{H}), 1.39$ (sextet, $J=7.3 \mathrm{~Hz}, 2 \mathrm{H}), 1.43-1.54(\mathrm{~m}, 2 \mathrm{H}), 1.91$ (quintet, $J=5.9 \mathrm{~Hz}$, 2H), $2.30(\mathrm{t}, J=5.9 \mathrm{~Hz}, 2 \mathrm{H}), 2.39(\mathrm{t}, J=7.3 \mathrm{~Hz}, 2 \mathrm{H}), 2.36-2.47(\mathrm{~m}, 2 \mathrm{H}), 3.79(\mathrm{~s}, 3 \mathrm{H}), 5.81$ $(\mathrm{s}, 1 \mathrm{H}), 6.84(\mathrm{~d}, J=8.8 \mathrm{~Hz}, 2 \mathrm{H}), 7.28(\mathrm{~d}, J=8.8 \mathrm{~Hz}, 2 \mathrm{H}) .{ }^{13} \mathrm{C} \mathrm{NMR}\left(\mathrm{CDCl}_{3}\right) \delta 13.99,22.45$, $22.63,26.99,27.05,27.10,30.04,30.15,38.84,55.27,102.42,105.93,113.24,113.72$, $127.25,129.63,149.37,158.40,176.90,203.14$. $[\alpha]^{20} \mathrm{D}+20.5\left(c 1.07, \mathrm{CHCl}_{3}\right)$ for $93 \%$ ee. HRMS (FAB) Calcd for $\mathrm{C}_{24} \mathrm{H}_{32} \mathrm{O}_{3}: 388.2350\left([\mathrm{M}]^{+}\right)$. Found: $388.2345\left([\mathrm{M}]^{+}\right)$. 3-(2Cyclohexyl-2-phenylethenylidene)-1-(trimethylacetoxy)cyclohexene (4bm): purified by PTLC $($ Hexane/AcOEt $=15 / 1)(91 \%$ yield $) ;{ }^{1} \mathrm{H}$ NMR $\left(\mathrm{CDCl}_{3}\right) \delta 1.08-1.24(\mathrm{~m}, 3 \mathrm{H}), 1.25(\mathrm{~s}$, 9H), 1.28-1.41 (m, 2H), 1.66-1.73 (m, 1H), 1.73-1.80 (m, 2H), 1.84-1.94 (m, 4H), $2.30(\mathrm{td}, J$ $=6.3,1.3 \mathrm{~Hz}, 2 \mathrm{H}), 2.37-2.49(\mathrm{~m}, 3 \mathrm{H}), 5.81(\mathrm{t}, J=1.3 \mathrm{~Hz}, 1 \mathrm{H}), 7.17(\mathrm{tt}, J=7.2,1.4 \mathrm{~Hz}, 1 \mathrm{H})$, $7.28(\mathrm{t}, J=7.2 \mathrm{~Hz}, 2 \mathrm{H}), 7.33(\mathrm{~d}, J=7.2 \mathrm{~Hz}, 2 \mathrm{H}) .{ }^{13} \mathrm{C} \mathrm{NMR}\left(\mathrm{CDCl}_{3}\right) \delta 22.62,26.29,26.58$, $26.60,27.03,27.08,32.97,33.12,38.57,38.81,103.24,112.92,113.10,126.42,126.62$, 128.24, 137.04, 149.37, 176.77, 203.39. $[\alpha]^{20} \mathrm{D}+8.8\left(c 1.05, \mathrm{CHCl}_{3}\right)$ for $70 \%$ ee. HRMS (FAB) Calcd for $\mathrm{C}_{25} \mathrm{H}_{32} \mathrm{O}_{2}: 364.2401\left([\mathrm{M}]^{+}\right)$. Found: $364.2410\left([\mathrm{M}]^{+}\right)$. 3-(2-Cyclohexyl-2(4-methoxyphenyl)ethenylidene)-1-(trimethylacetoxy)cyclohexene (4bo): purified by PTLC $($ Hexane/AcOEt $=10 / 1)(80 \%$ yield $) ;{ }^{1} \mathrm{H}$ NMR $\left(\mathrm{CDCl}_{3}\right) \delta 1.05-1.41(\mathrm{~m}, 5 \mathrm{H}), 1.25(\mathrm{~s}, 9 \mathrm{H})$, 1.64-1.72 (m, 1H), 1.71-1.79 (m, 2H), 1.82-1.95 (m, 4H), $2.29(\mathrm{td}, J=6.2,1.1 \mathrm{~Hz}, 2 \mathrm{H}), 2.35-$ $2.47(\mathrm{~m}, 3 \mathrm{H}), 3.80(\mathrm{~s}, 3 \mathrm{H}), 5.80(\mathrm{t}, J=1.1 \mathrm{~Hz}, 1 \mathrm{H}), 6.83(\mathrm{~d}, J=8.9 \mathrm{~Hz}, 2 \mathrm{H}), 7.25(\mathrm{~d}, J=8.9$ $\mathrm{Hz}, 2 \mathrm{H}) .{ }^{13} \mathrm{C} \mathrm{NMR}\left(\mathrm{CDCl}_{3}\right) \delta 22.65,26.33,26.62,26.63,27.05,27.10,27.17,32.96,33.13$, $38.71,38.84,55.25,103.14,112.39$, 113.37, 113.76, 127.67, 129.29, 149.23, 158.36, 176.85, 202.92. $[\alpha]^{20} \mathrm{D}+14.9\left(c 1.01, \mathrm{CHCl}_{3}\right)$ for $80 \%$ ee. HRMS (FAB) Calcd for $\mathrm{C}_{26} \mathrm{H}_{35} \mathrm{O}_{3}$ : 
$395.2586\left([\mathrm{M}+\mathrm{H}]^{+}\right) . \quad$ Found: $395.2573\left([\mathrm{M}+\mathrm{H}]^{+}\right) . \quad 3$ - $(\mathbf{3}, \mathbf{3}$ - D i methyl-2 phenylbutenylidene)-1-(trimethylacetoxy)cyclohexene $(4 \mathrm{~cm})$ : purified by PTLC $($ Hexane/AcOEt $=20 / 1)(44 \%$ yield $) ;{ }^{1} \mathrm{H} \mathrm{NMR}\left(\mathrm{CDCl}_{3}\right) \delta 1.12(\mathrm{~s}, 9 \mathrm{H}), 1.24(\mathrm{~s}, 9 \mathrm{H}), 1.83$ (quintet, $J=6.3 \mathrm{~Hz}, 2 \mathrm{H}), 2.16-2.28(\mathrm{~m}, 2 \mathrm{H}), 2.30-2.41(\mathrm{~m}, 2 \mathrm{H}), 5.79(\mathrm{t}, J=1.4 \mathrm{~Hz}, 1 \mathrm{H})$, 7.19-7.24 (m, 3H), 7.24-7.29 (m, 2H). ${ }^{13} \mathrm{C} \mathrm{NMR}\left(\mathrm{CDCl}_{3}\right) \delta$ 22.41, 27.01, 27.07, 27.29, 30.07, 35.14, 38.84, 99.94, 113.67, 116.59, 126.40, 127.67, 129.38, 138.05, 148.85, 176.81, 201.18. $[\alpha]^{20} \mathrm{D}-8.8\left(c 1.00, \mathrm{CHCl}_{3}\right)$ for $26 \%$ ee. HRMS (FAB) Calcd for $\mathrm{C}_{23} \mathrm{H}_{30} \mathrm{O}_{2}: 338.2246\left([\mathrm{M}]^{+}\right)$.

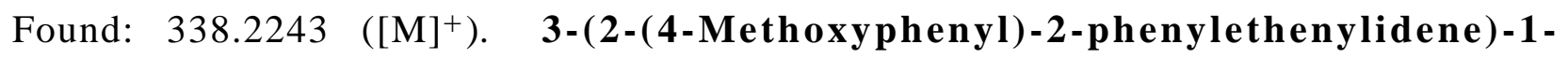
(trimethylacetoxy)cyclohexene $(\mathbf{4 d m})$ : purified by PTLC $($ Hexane/AcOEt $=7 / 1)(56 \%$ yield); ${ }^{1} \mathrm{H} \mathrm{NMR}\left(\mathrm{CDCl}_{3}\right) \delta 1.25(\mathrm{~s}, 9 \mathrm{H}), 1.93$ (quintet, $\left.J=6.2 \mathrm{~Hz}, 2 \mathrm{H}\right), 2.31(\mathrm{td}, J=6.2,1.3$ $\mathrm{Hz}, 2 \mathrm{H}), 2.52(\mathrm{t}, J=6.2 \mathrm{~Hz}, 2 \mathrm{H}), 3.81(\mathrm{~s}, 3 \mathrm{H}), 5.92(\mathrm{t}, J=1.3 \mathrm{~Hz}, 1 \mathrm{H}), 6.86(\mathrm{~d}, J=8.9 \mathrm{~Hz}$, 2H), 7.21-7.28 (m, 1H), $7.23(\mathrm{~d}, J=8.9 \mathrm{~Hz}, 2 \mathrm{H}), 7.28-7.34(\mathrm{~m}, 4 \mathrm{H}) .{ }^{13} \mathrm{C} \mathrm{NMR}\left(\mathrm{CDCl}_{3}\right) \delta$ $13.72,22.17,22.32,26.59,26.77,26.83,29.70,29.77,38.57,102.28,106.19,112.70$, $125.89,126.22,127.98,137.07,149.25,176.58,203.39 .[\alpha]^{20} \mathrm{D}-1.7\left(c 1.00, \mathrm{CHCl}_{3}\right)$ for $75 \%$ ee. HRMS (FAB) Calcd for $\mathrm{C}_{26} \mathrm{H}_{28} \mathrm{O}_{3}: 388.2037\left([\mathrm{M}]^{+}\right)$. Found: $388.2039\left([\mathrm{M}]^{+}\right)$. 3-(2Phenylhexenylidene)-1-(trimethylacetoxy)cyclopentene (4em): purified by short silica gel column (Hexane) (80\% yield, contaminated with ca. $20 \%$ of dienyl ketone); ${ }^{1} \mathrm{H}$ NMR $\left(\mathrm{CDCl}_{3}\right)$ $\delta 0.92(\mathrm{t}, J=7.5 \mathrm{~Hz}, 3 \mathrm{H}), 1.27(\mathrm{~s}, 9 \mathrm{H}), 1.34-1.44(\mathrm{~m}, 2 \mathrm{H}), 1.47-1.55(\mathrm{~m}, 2 \mathrm{H}), 2.44(\mathrm{t}, J=7.5$ $\mathrm{Hz}, 2 \mathrm{H}), 2.67-2.84(\mathrm{~m}, 4 \mathrm{H}), 5.98(\mathrm{t}, J=1.7 \mathrm{~Hz}, 1 \mathrm{H}), 7.17(\mathrm{tt}, J=7.4,1.2 \mathrm{~Hz}, 1 \mathrm{H}), 7.29$ (t, $J=$ $7.4 \mathrm{~Hz}, 2 \mathrm{H}), 7.38(\mathrm{~d}, J=7.4 \mathrm{~Hz}, 2 \mathrm{H}) .{ }^{13} \mathrm{C} \mathrm{NMR}\left(\mathrm{CDCl}_{3}\right) \delta 13.97,22.40,25.49,26.97,30.04$, 30.29 , 30.66, 39.12, 108.88, 109.97, 113.57, 126.16, 126.36, 128.15, 137.83, 154.08, 175.59, 199.92. $[\alpha]^{20} \mathrm{D}-10.8\left(c 1.00, \mathrm{CHCl}_{3}\right)$ for $70 \%$ ee. HRMS (FAB) Calcd for $\mathrm{C}_{22} \mathrm{H}_{28} \mathrm{O}_{2}$ : $324.2089\left([\mathrm{M}]^{+}\right)$. Found: $324.2087\left([\mathrm{M}]^{+}\right)$.

Conversion of Silyl Enol Ether 3am into Triflate 5am. In a similar manner to the procedure of conversion of silyl enol ether $\mathbf{3}$ into pivalate $\mathbf{4}$, the lithium enolate was generated from 3am by treatment with methyllithium. To the solution was added a solution of $N$-(2-pyridyl)triflimide $(215 \mathrm{mg}, 0.60 \mathrm{mmol})$ in THF $(1.0 \mathrm{~mL})$ at $-78^{\circ} \mathrm{C}$ and the mixture was stirred ar room temperature for $12 \mathrm{~h}$. The reaction was quenched with $\mathrm{H}_{2} \mathrm{O}$ (ca. $0.5 \mathrm{~mL}$ ). The 
solvent was removed in vacuo, and the residue was diluted with $\mathrm{Et}_{2} \mathrm{O}$. The mixture was washed with $\mathrm{H}_{2} \mathrm{O}$, before it was dried over $\mathrm{MgSO}_{4}$ and concentrated under reduced pressure. The crude product was purified by PTLC $($ Hexane/AcOEt $=20 / 1)$ to give triflate 5am $(94.2$ mg, $82 \%$ yield). The enantiomeric excess of 5am was determined by HPLC analysis with a chiral stationary phase column (Daicel Chiralcel OD-H, eluent: Hexane). 3-(2Phenylhexenylidene)-1-(trifluoromethanesulfonyloxy)cyclohexene $\quad(5 \mathrm{am}):{ }^{1} \mathrm{H}$ $\operatorname{NMR}\left(\mathrm{CDCl}_{3}\right) \delta 0.93(\mathrm{t}, J=7.2 \mathrm{~Hz}, 3 \mathrm{H}), 1.40$ (sextet, $\left.J=7.2 \mathrm{~Hz}, 2 \mathrm{H}\right), 1.44-1.54(\mathrm{~m}, 2 \mathrm{H})$, 1.96 (quintet, $J=6.9 \mathrm{~Hz}, 2 \mathrm{H}), 2.36-2.47(\mathrm{~m}, 2 \mathrm{H}), 2.46(\mathrm{t}, J=7.2 \mathrm{~Hz}, 2 \mathrm{H}), 2.48(\mathrm{td}, J=6.2$, $1.4 \mathrm{~Hz}, 2 \mathrm{H}), 6.15(\mathrm{t}, J=1.4 \mathrm{~Hz}, 1 \mathrm{H}), 7.21(\mathrm{tt}, J=6.7,1.9 \mathrm{~Hz}, 1 \mathrm{H}), 7.27-7.37(\mathrm{~m}, 4 \mathrm{H})$. ${ }^{13} \mathrm{C} \mathrm{NMR}\left(\mathrm{CDCl}_{3}\right) \delta 13.92,22.35,22.49,26.40,27.83,29.93,101.11,107.62,117.75$, $118.86(\mathrm{q}, J=319.4 \mathrm{~Hz}), 126.31,127.05,128.44,136.45,148.48,205.00 . \quad[\alpha]^{20} \mathrm{D}+16.0$ (c $1.00, \mathrm{CHCl}_{3}$ ) for $92 \%$ ee. HRMS (FAB) Calcd for $\mathrm{C}_{19} \mathrm{H}_{22} \mathrm{O}_{3} \mathrm{SF}_{3}: 387.1242\left([\mathrm{M}+\mathrm{H}]^{+}\right)$. Found: $387.1234\left([\mathrm{M}+\mathrm{H}]^{+}\right)$.

\section{References}

(1) Cramer, R. Inorg. Synth. 1974, 15, 16.

(2) Comins, D. L.; Dehghani, A. Tetrahedron Lett. 1992, 33, 6299.

(3) Yoshida, K.; Hayashi, T. J. Am. Chem. Soc. 2003, 125, 2872.

(4) Hayashi, T.; Tokunaga, N.; Yoshida, K.; Han, J. W. J. Am. Chem. Soc. 2002, 124, 12102.

(5) Fu, X.; Zhang, S.; Yin, J.; Schumacher, D. P. Tetrahedron Lett. 2002, 43, 6673.

(6) Rathjen, H.-J.; Margaretha, P.; Wolff, S.; Agosta, W. C. J. Am. Chem. Soc. 1991, 113, 3904. 

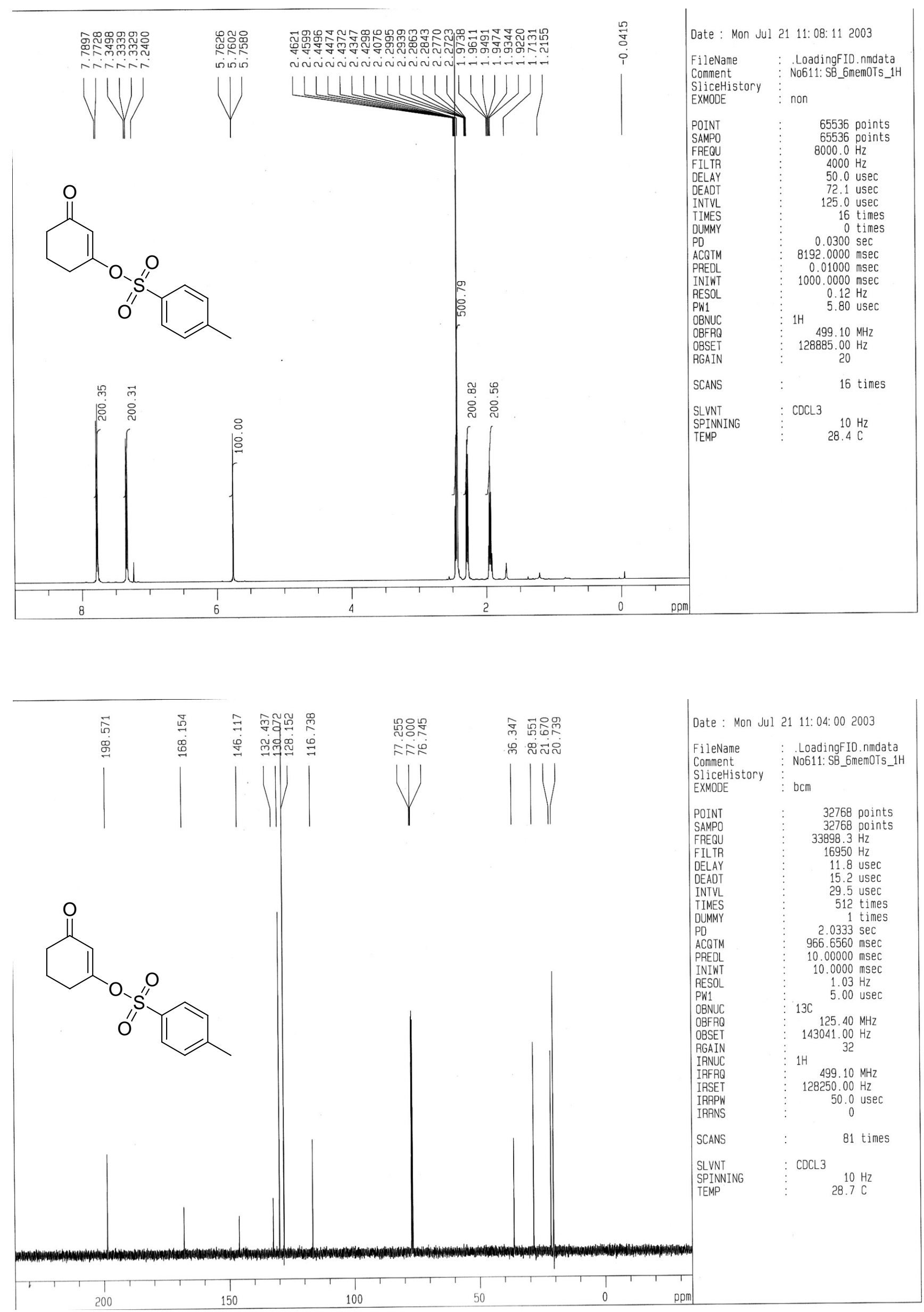

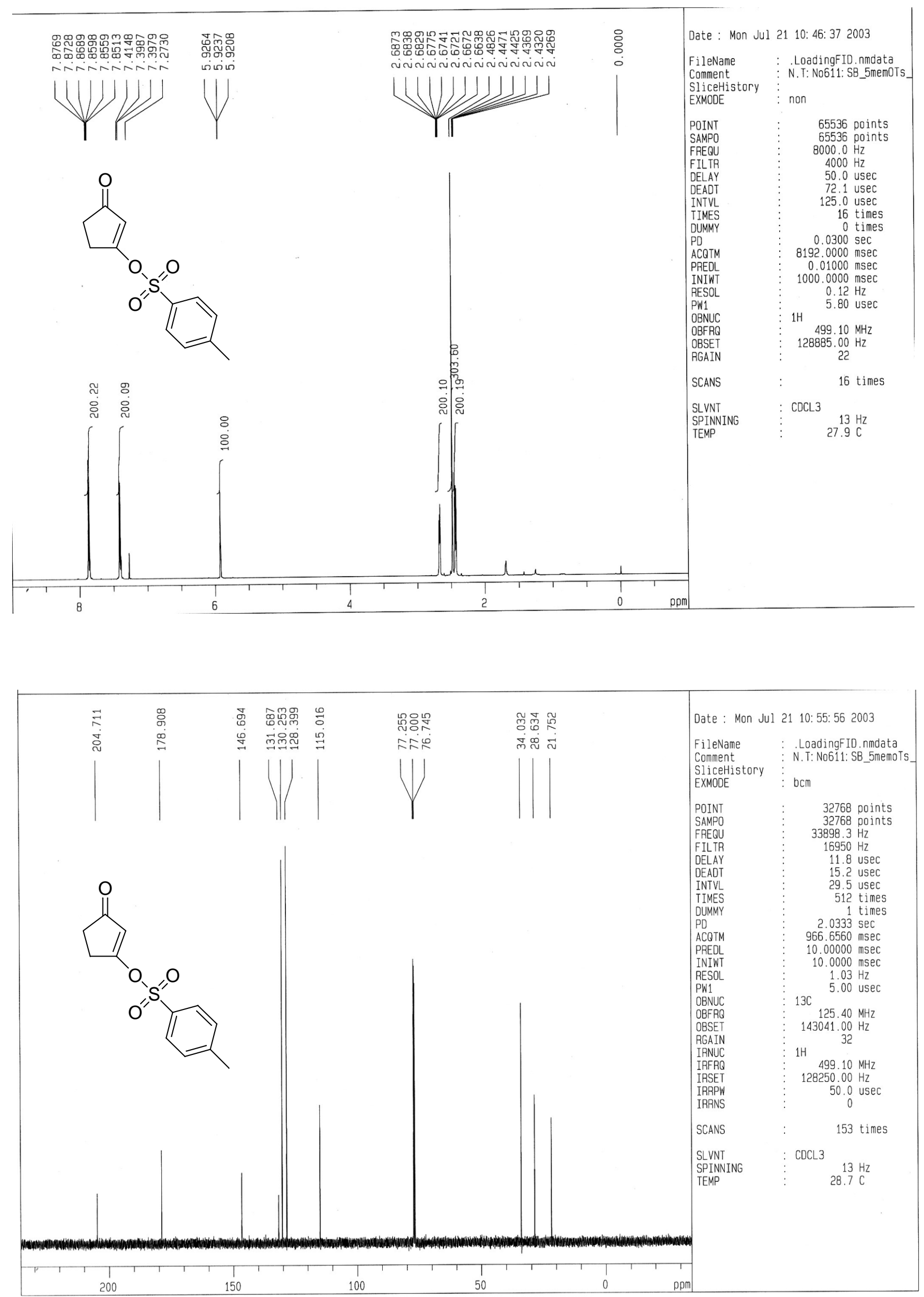

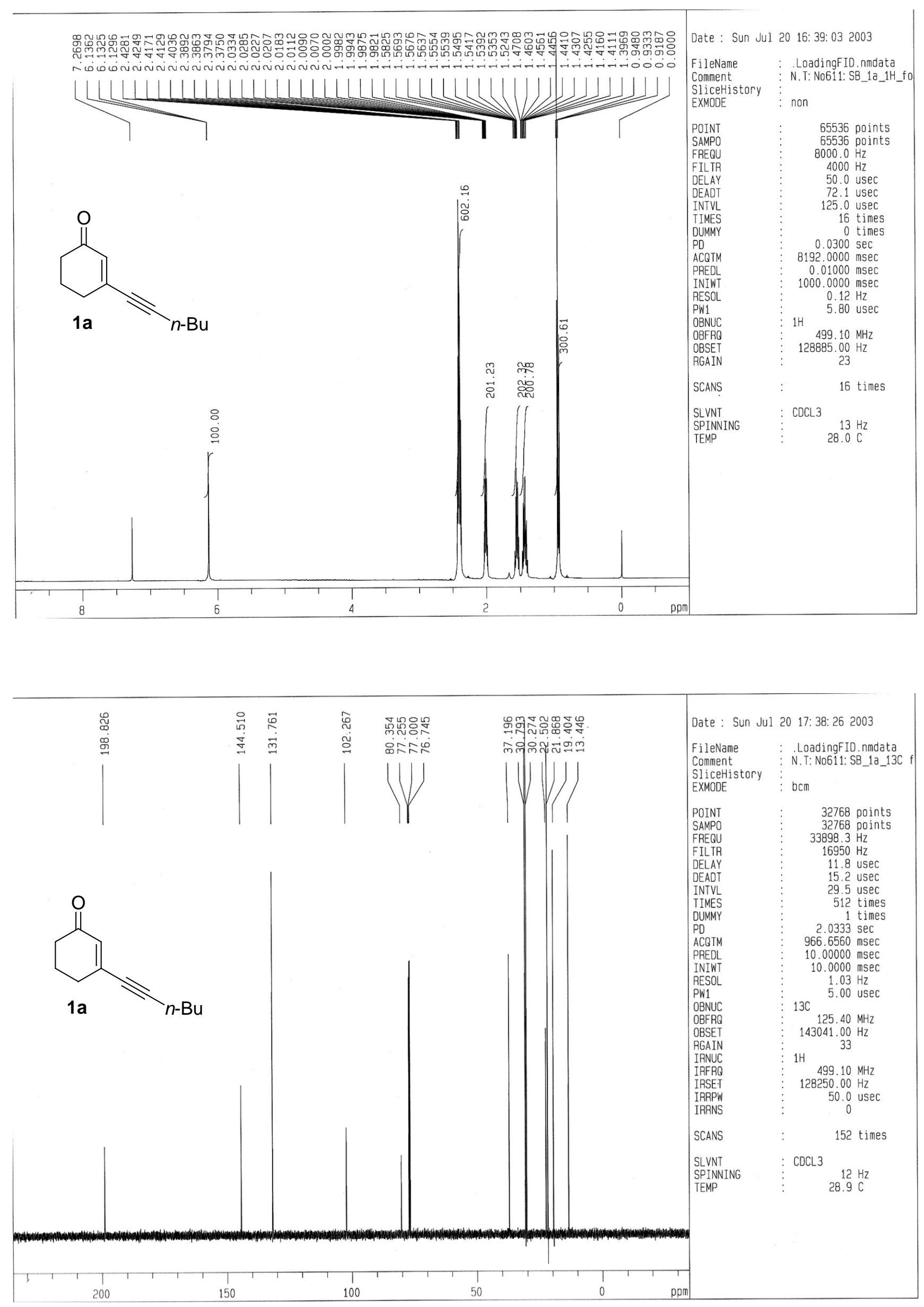

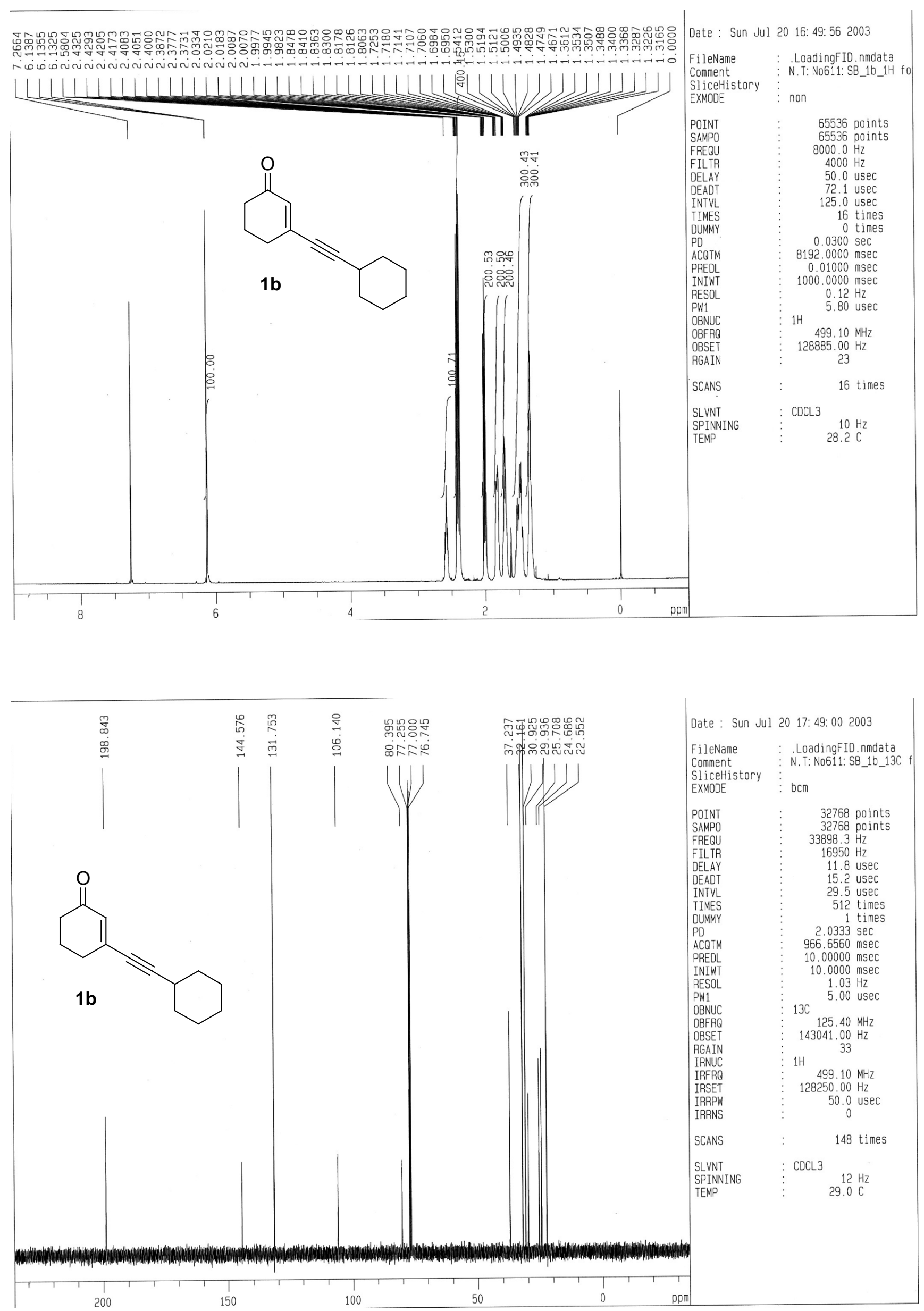

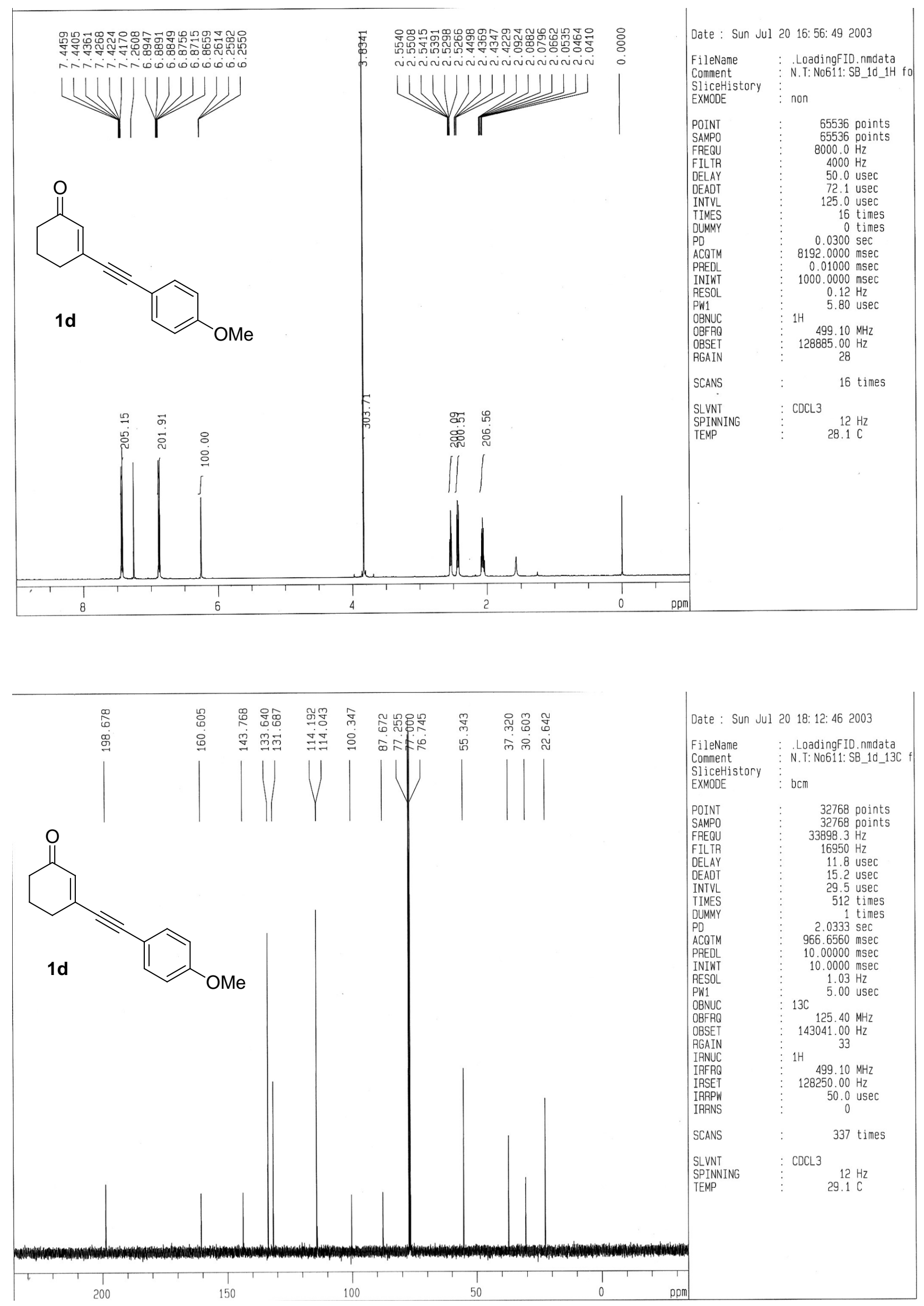


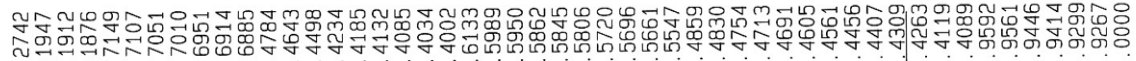
-
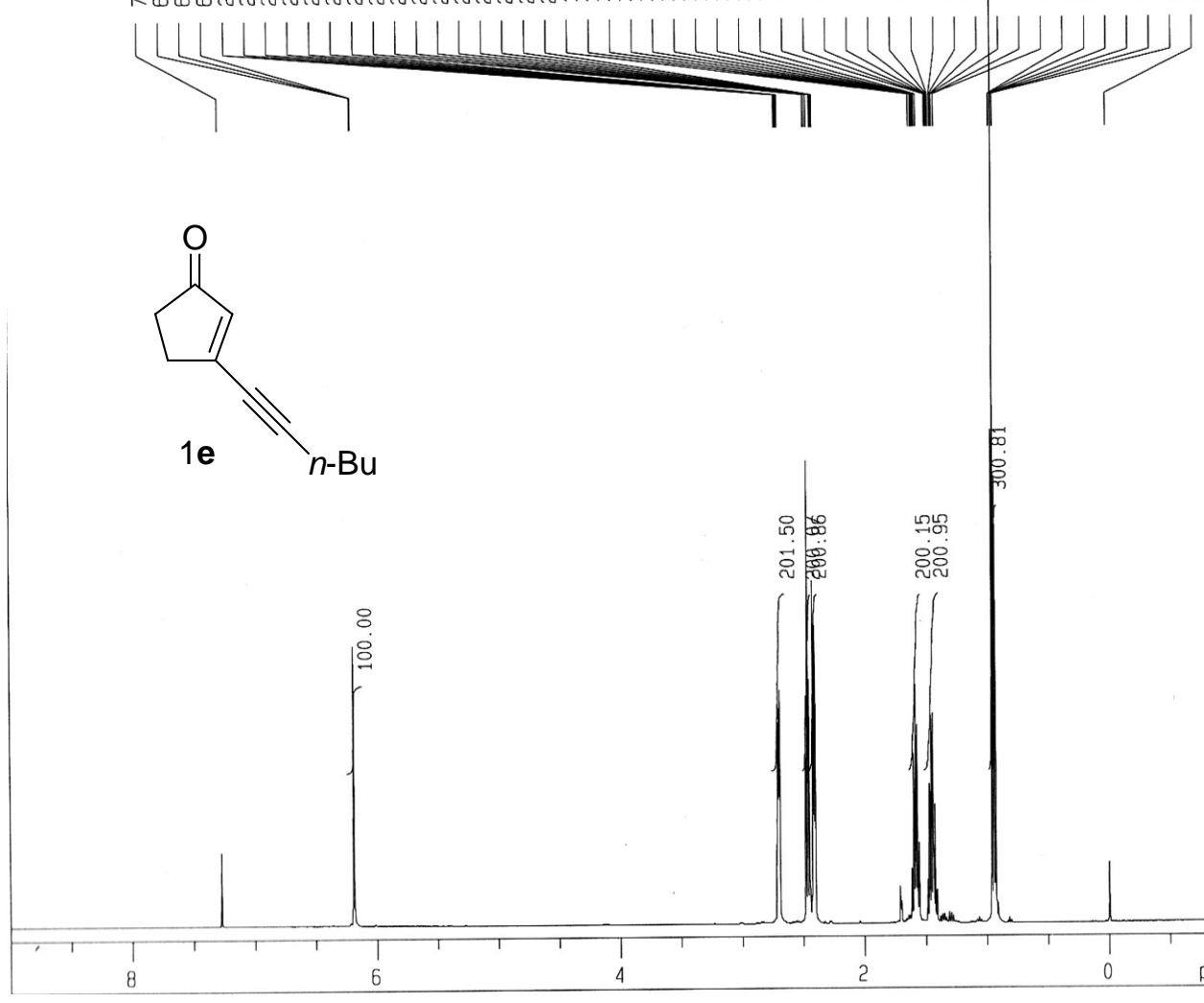
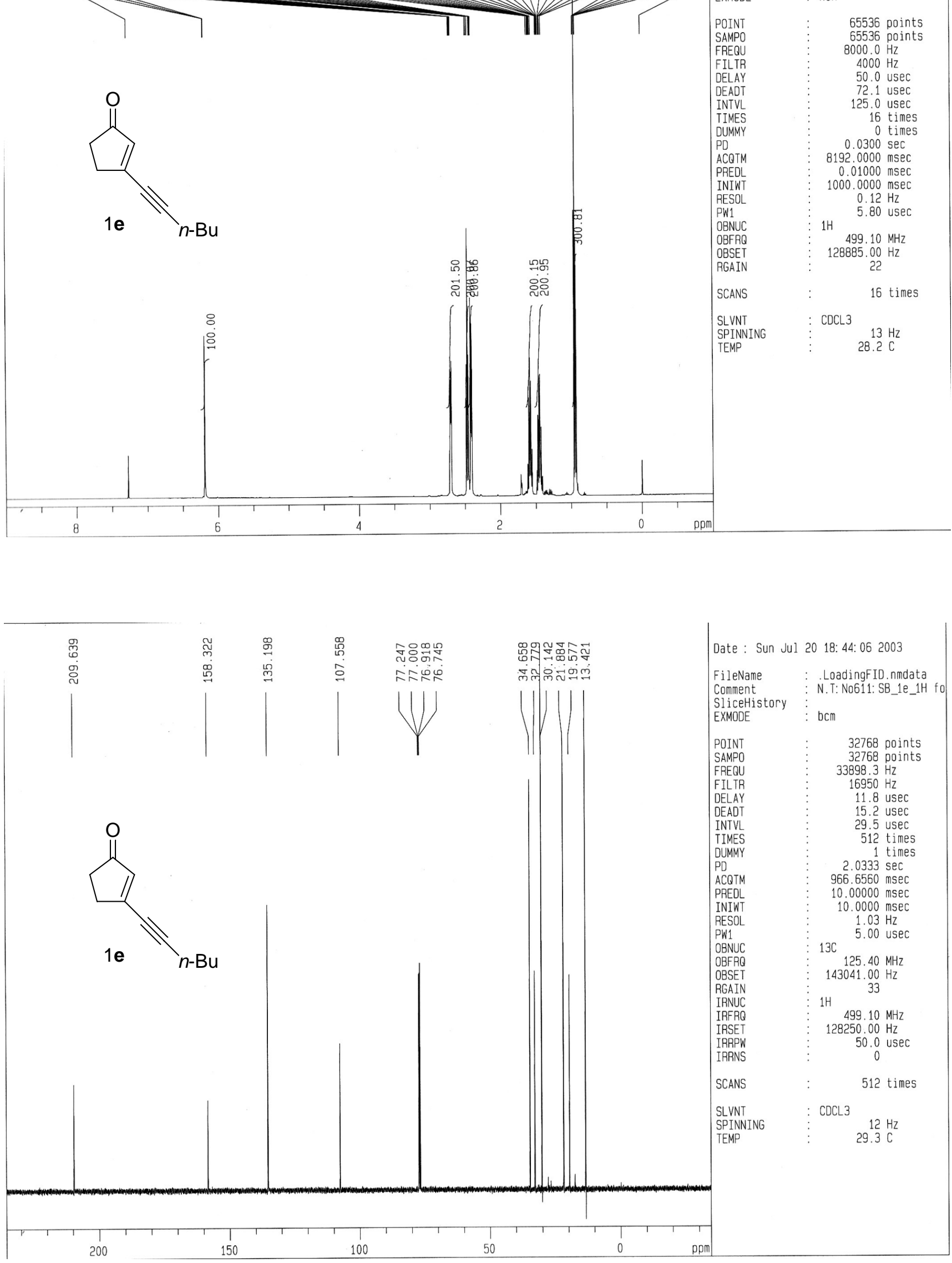

Date: Sun Jul 20 17: 10: 352003

FileNane $\quad:$ LoadingFID nmdata Comment : N.T: No611:SB_1e_1H fo SliceHistory EXMODE non

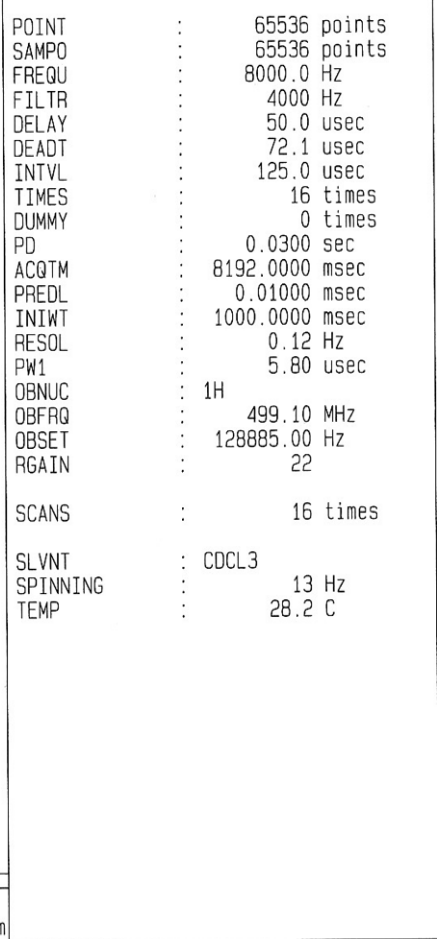



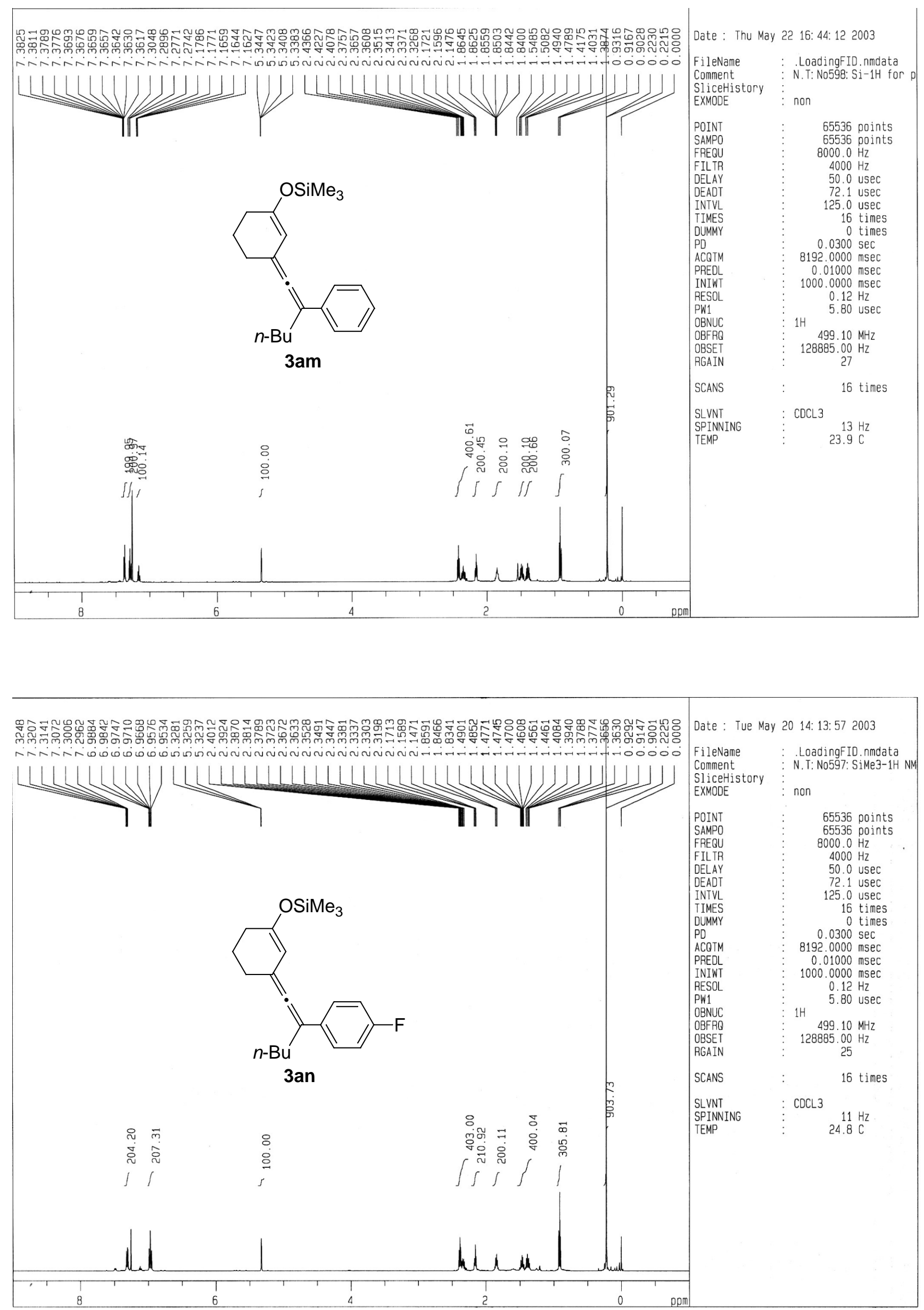

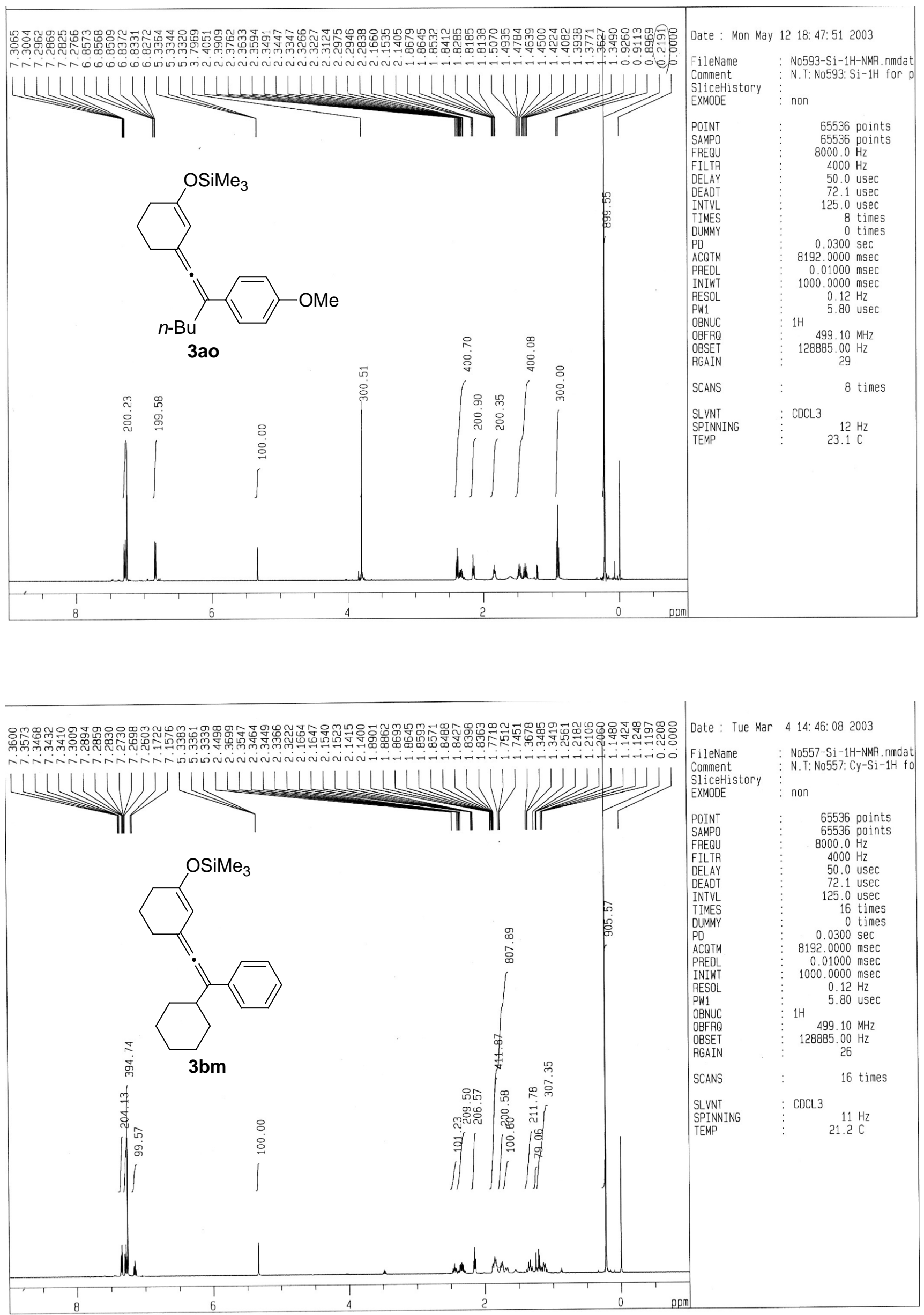


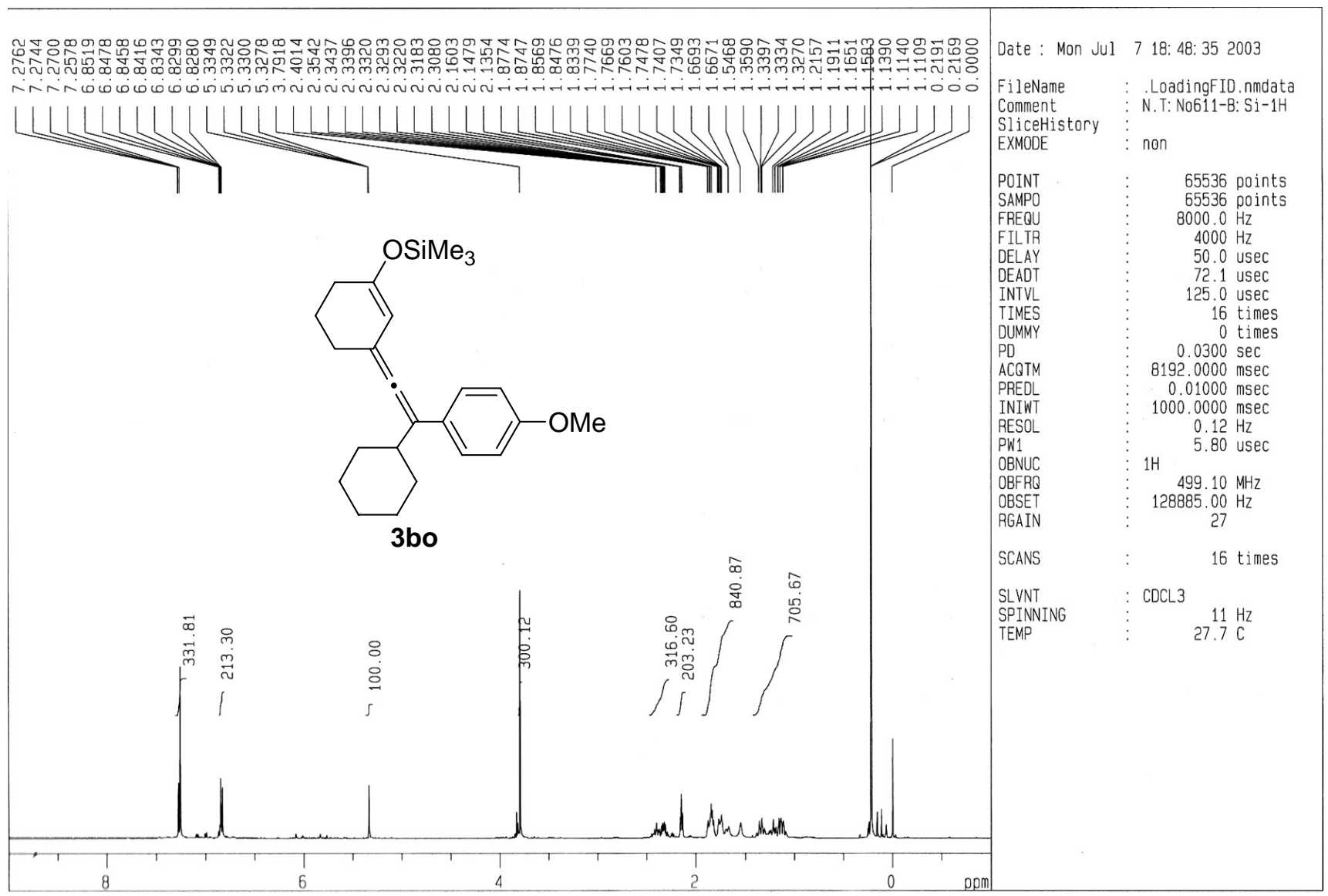

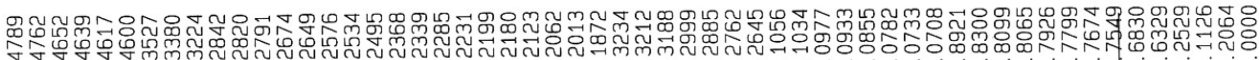

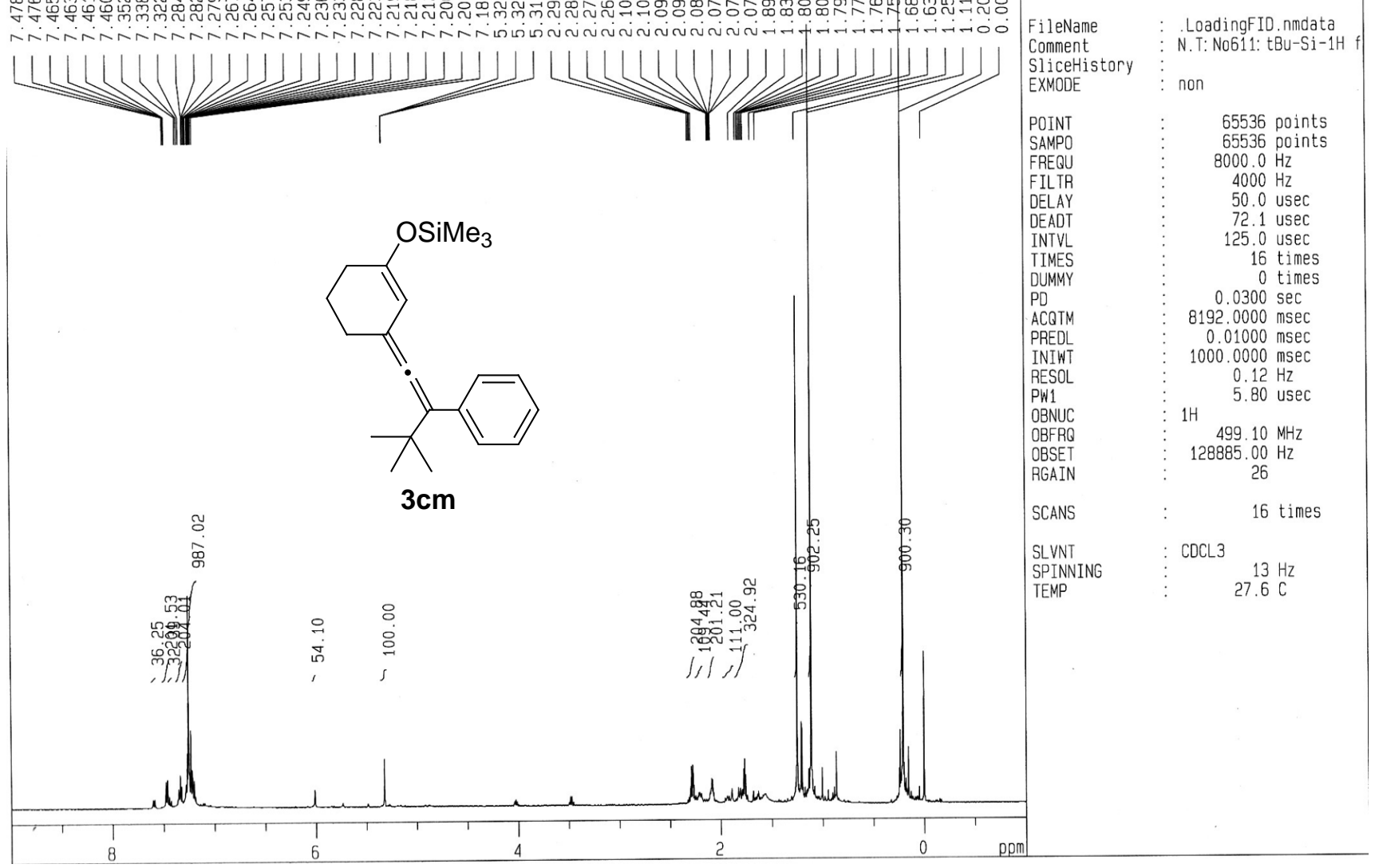



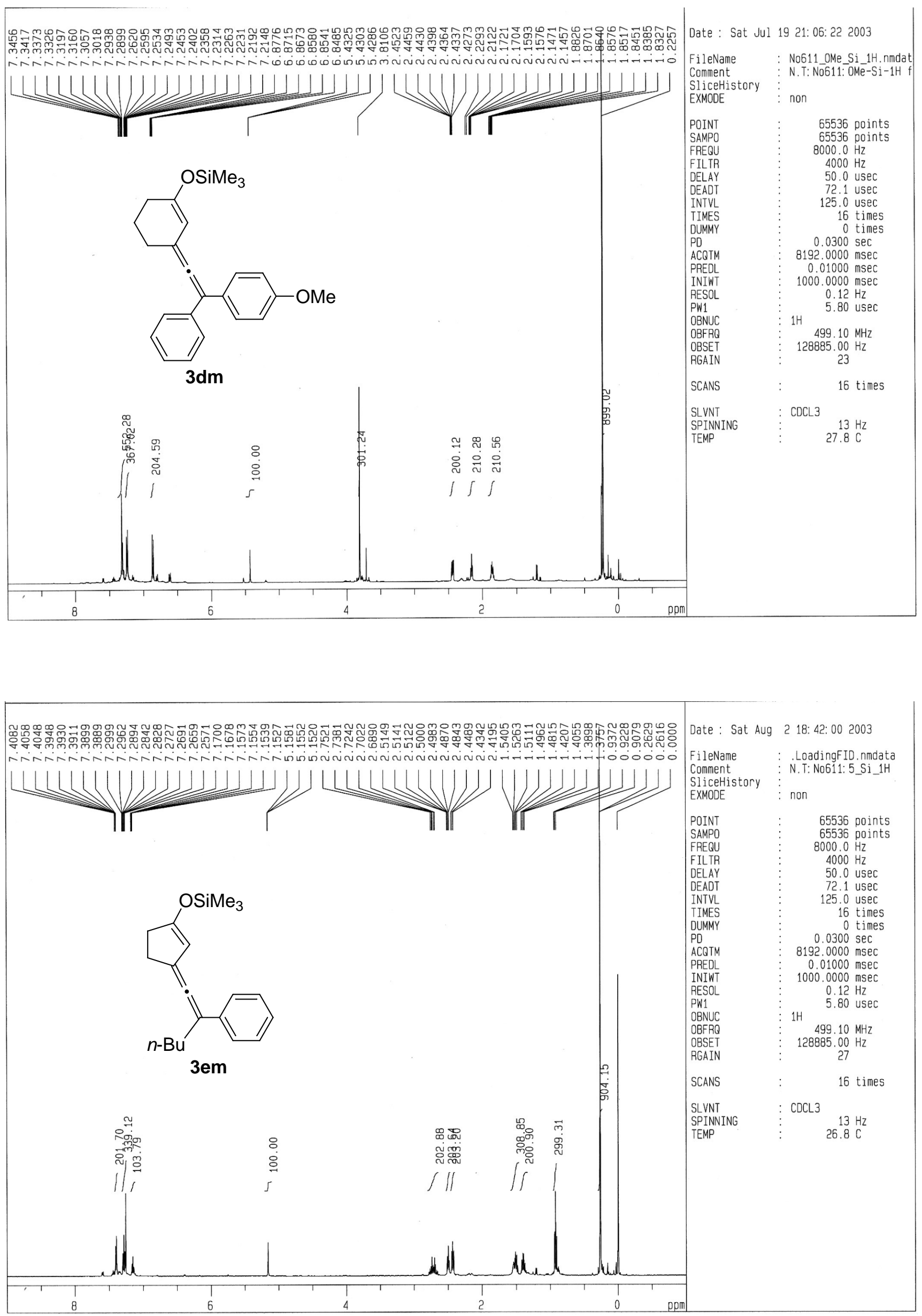

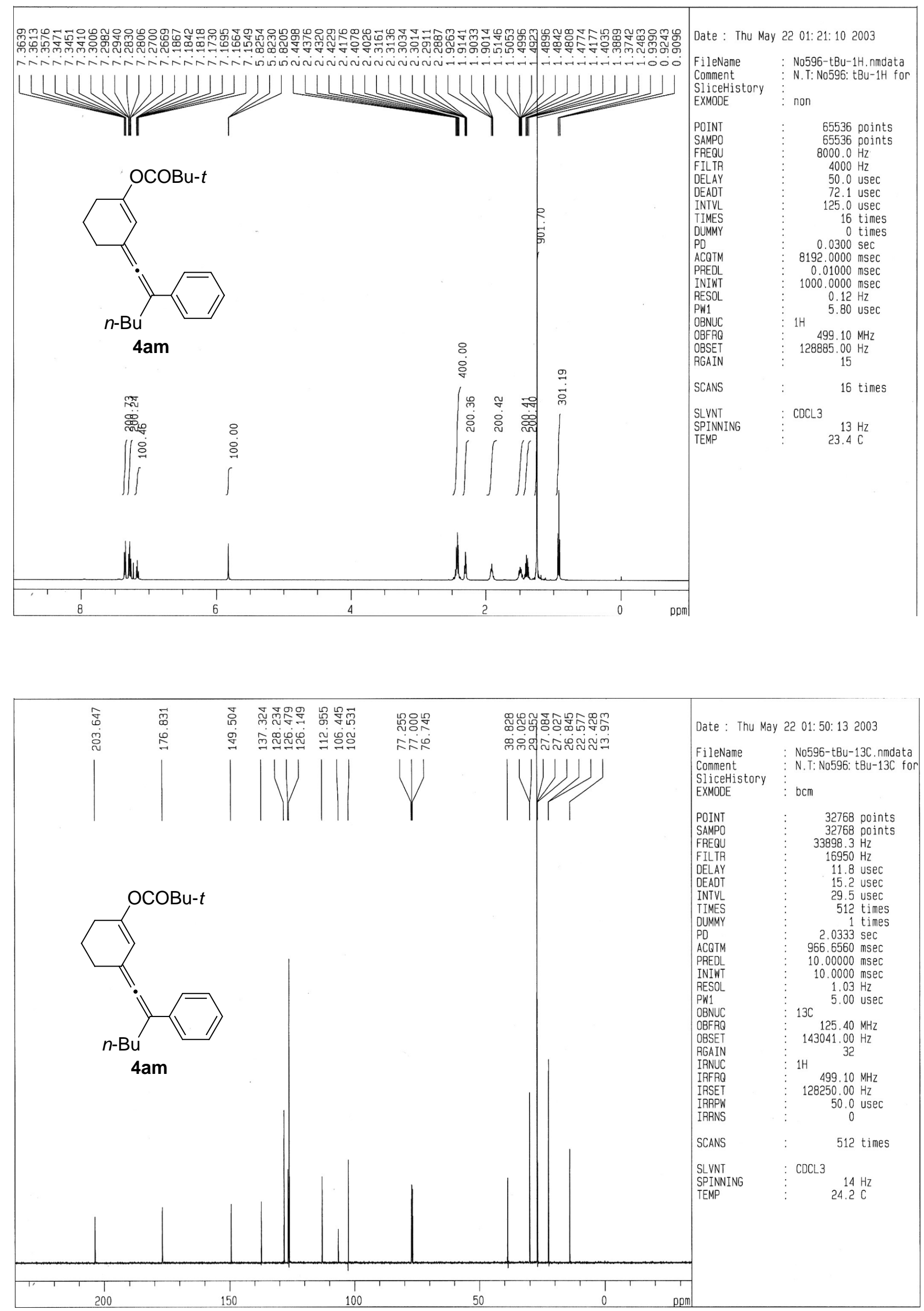

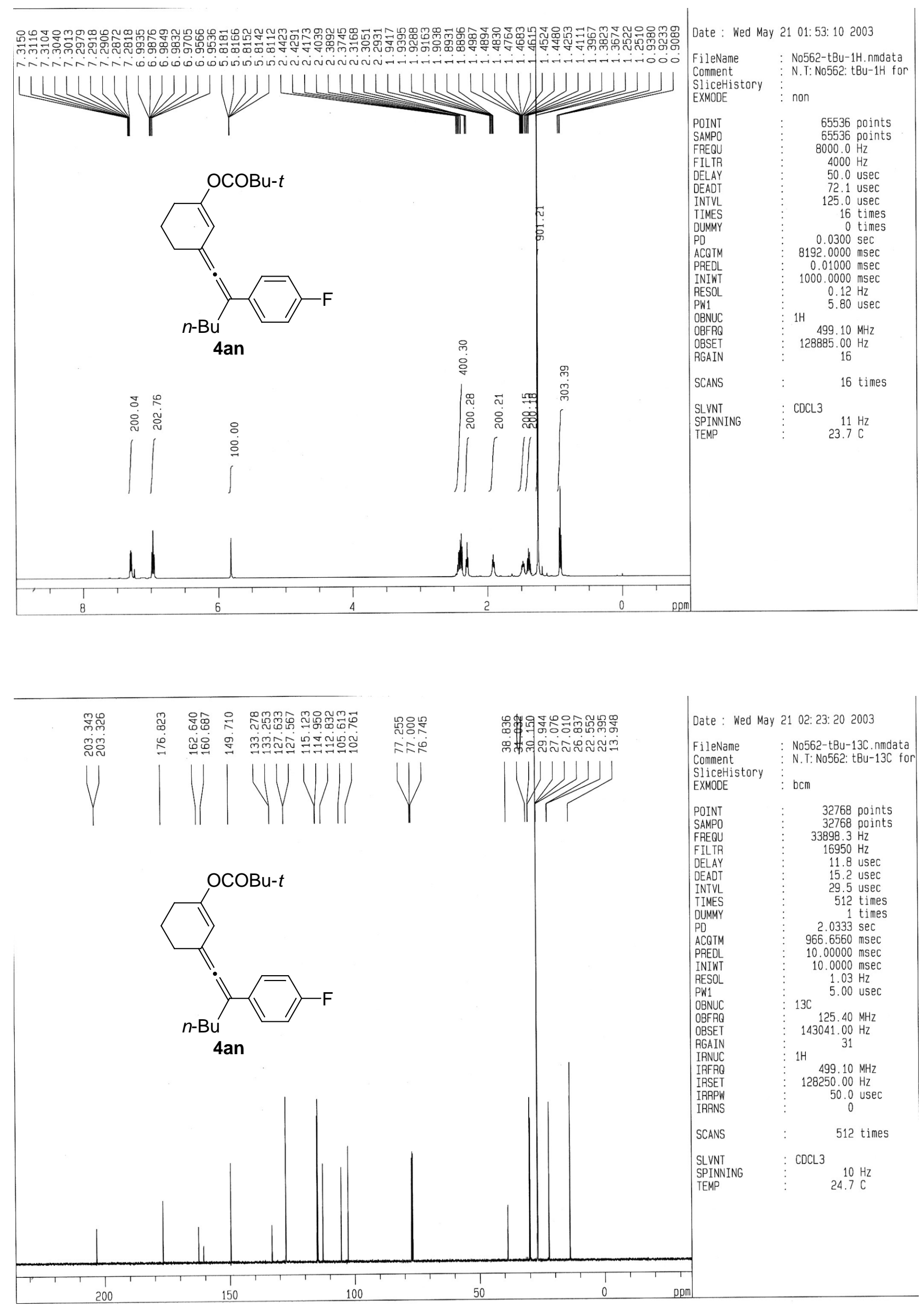

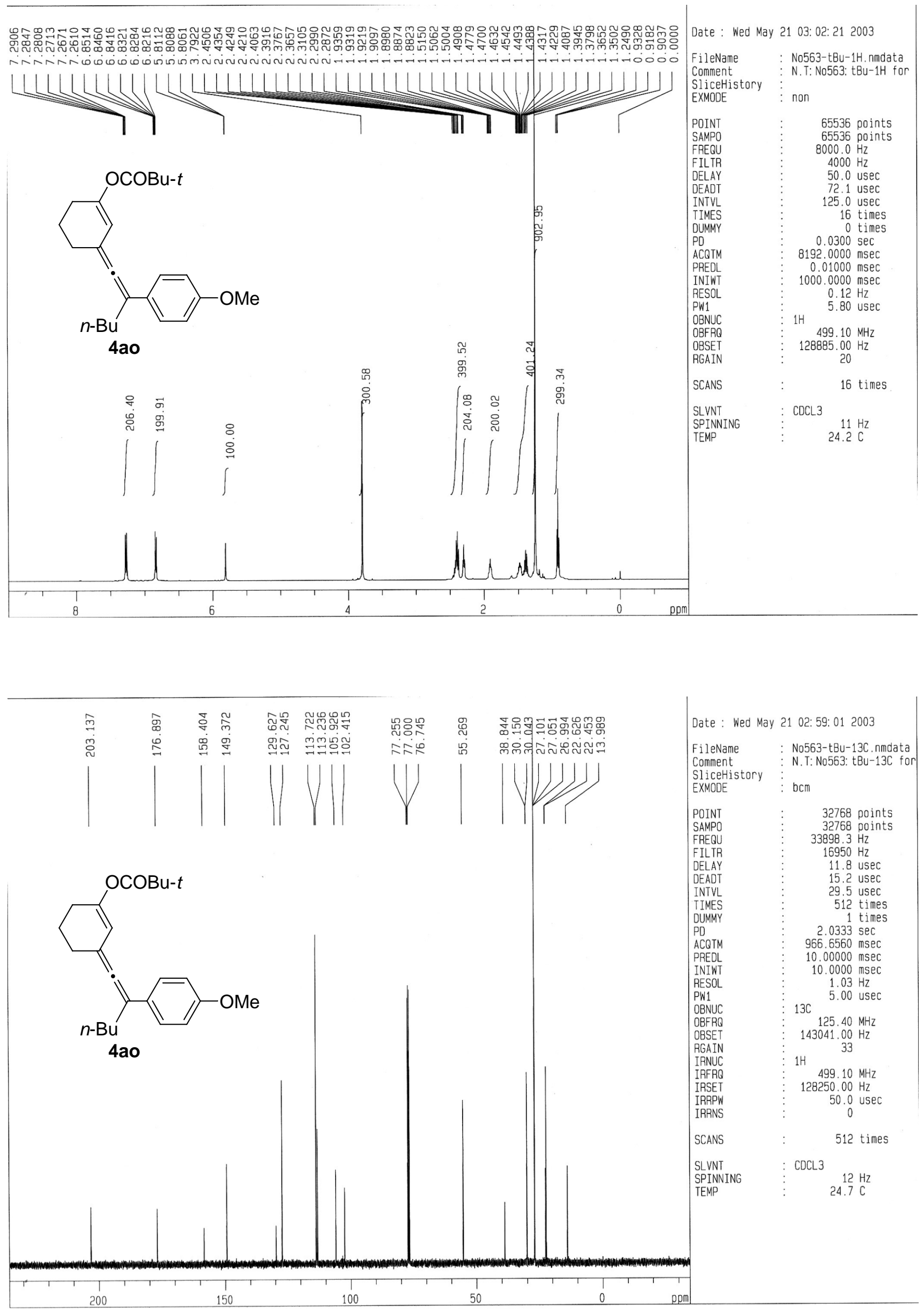

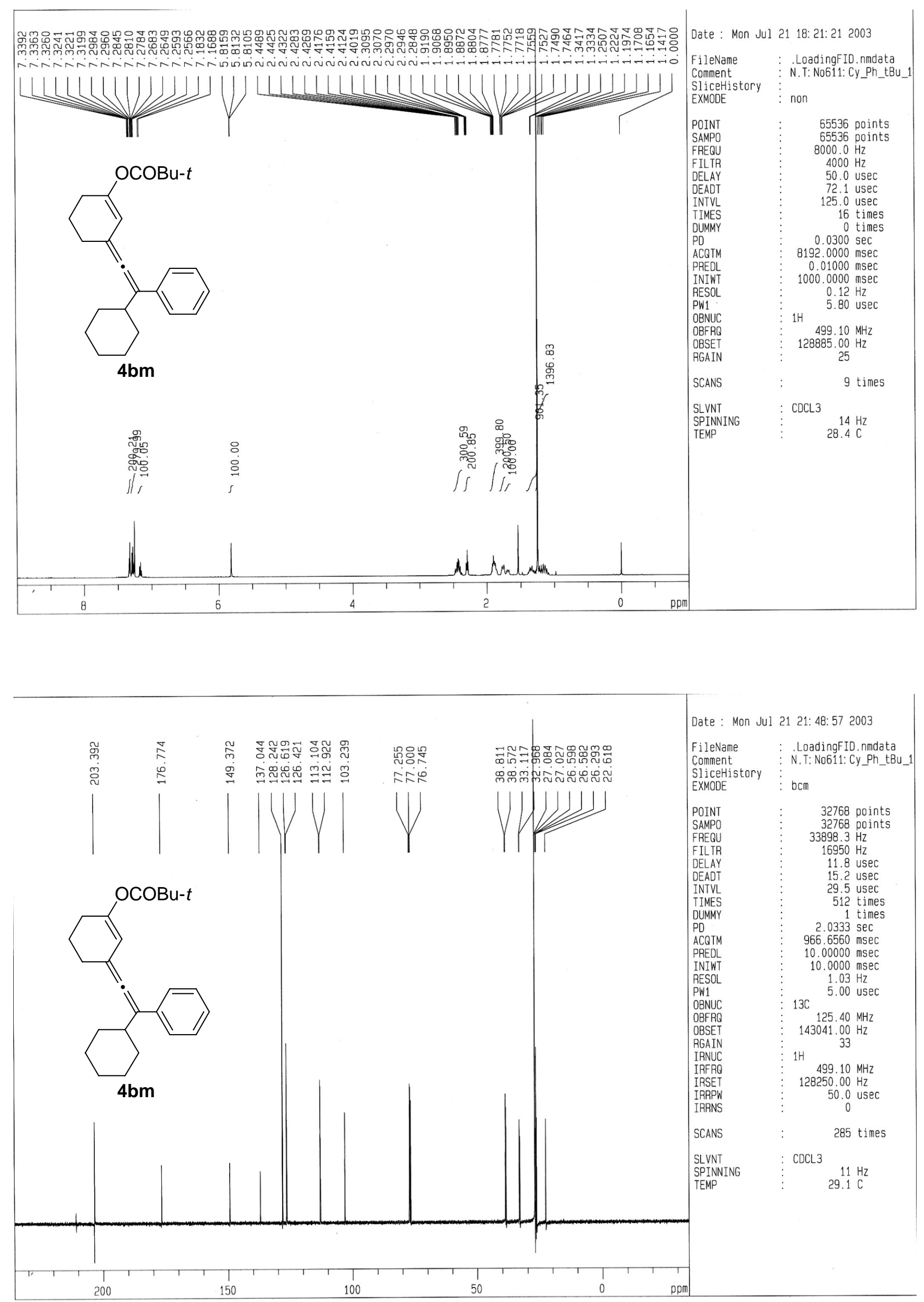

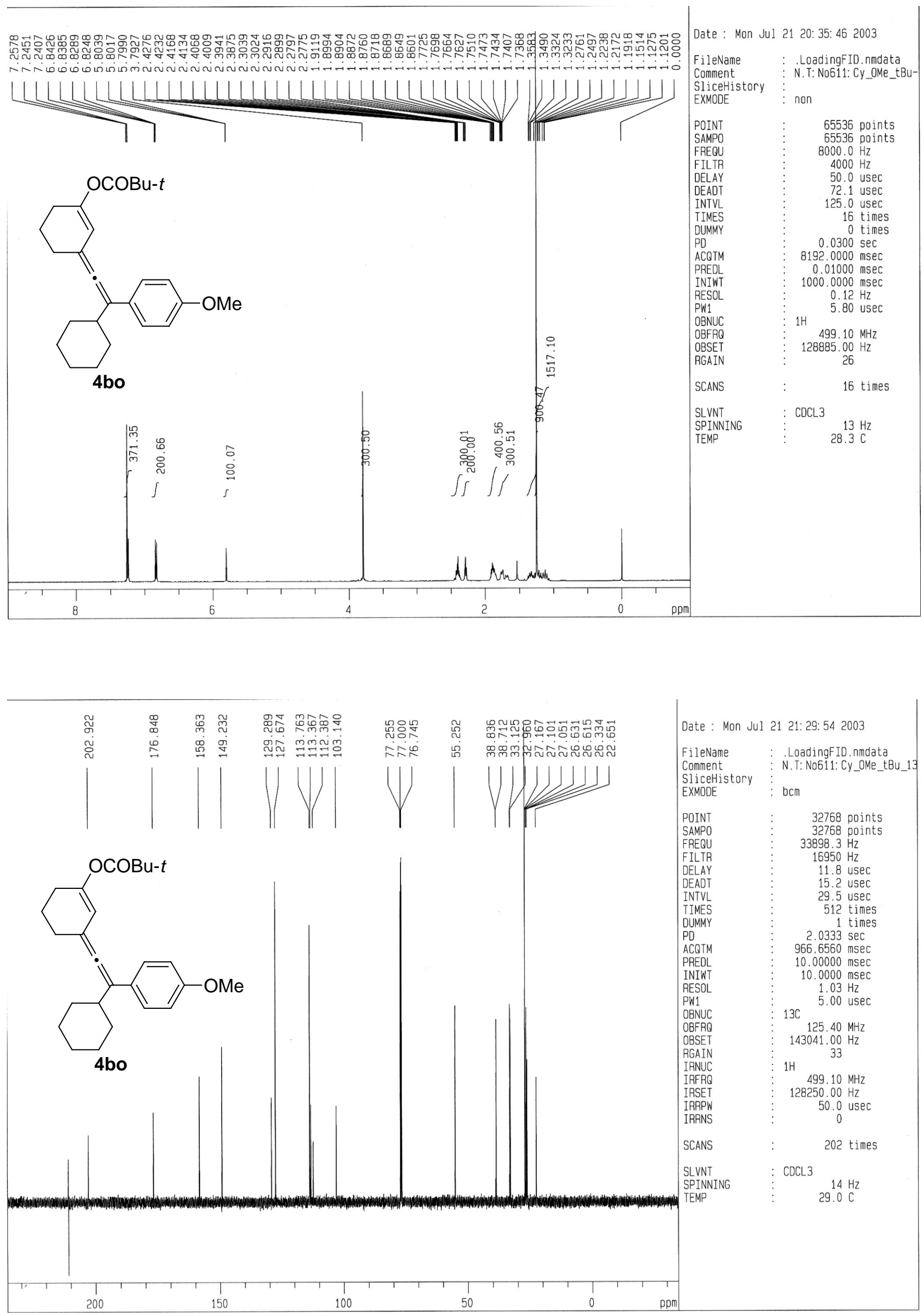

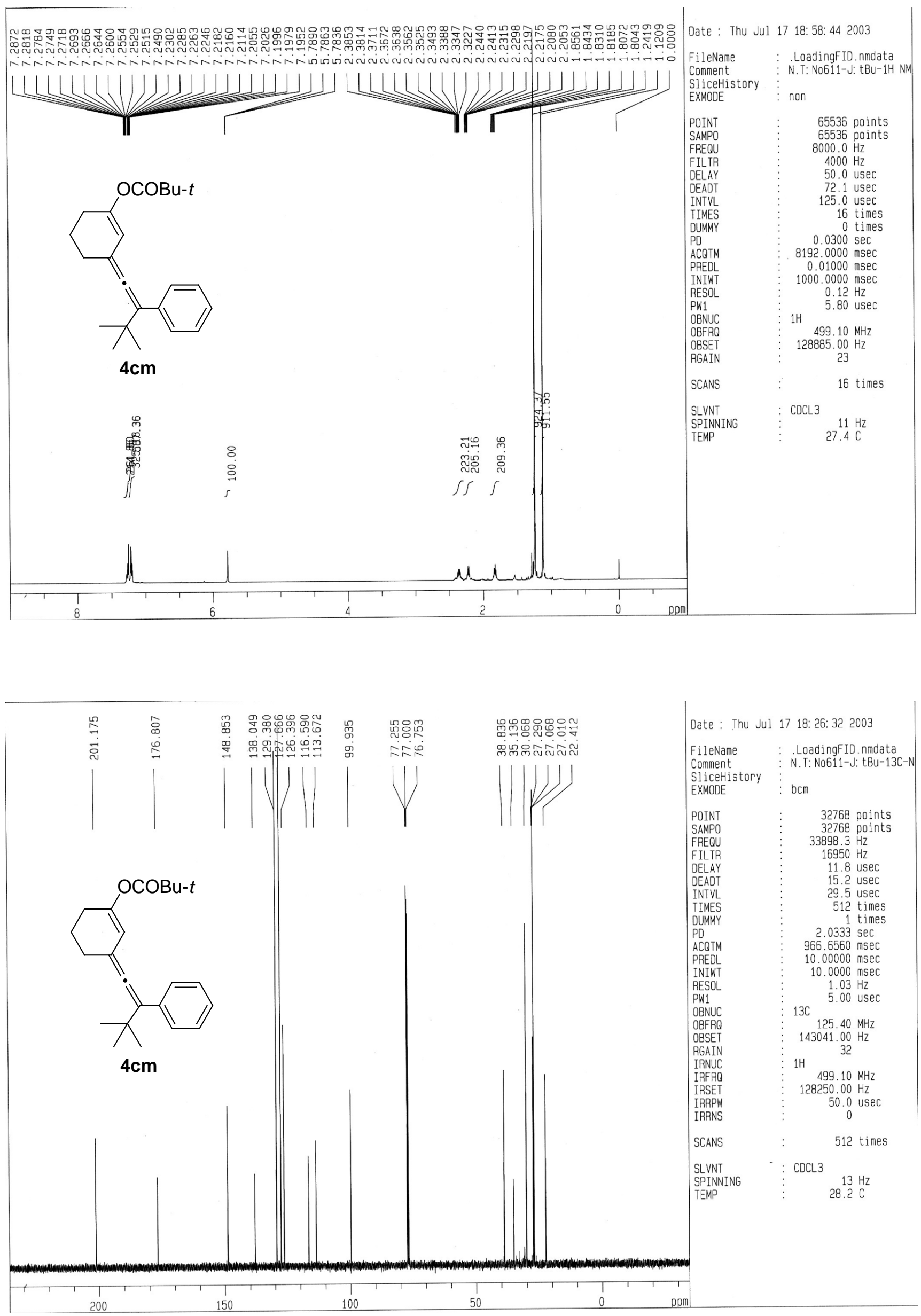

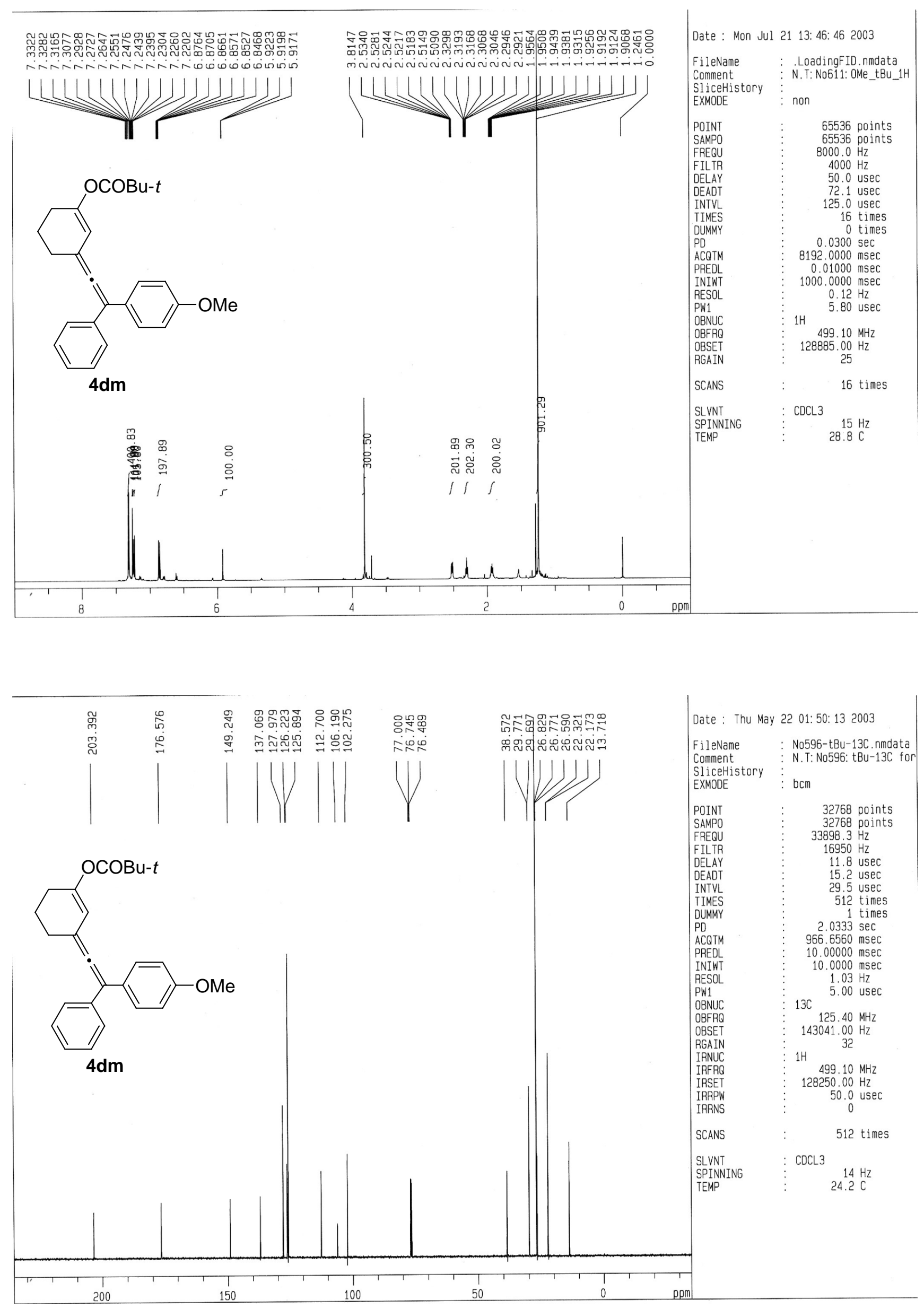

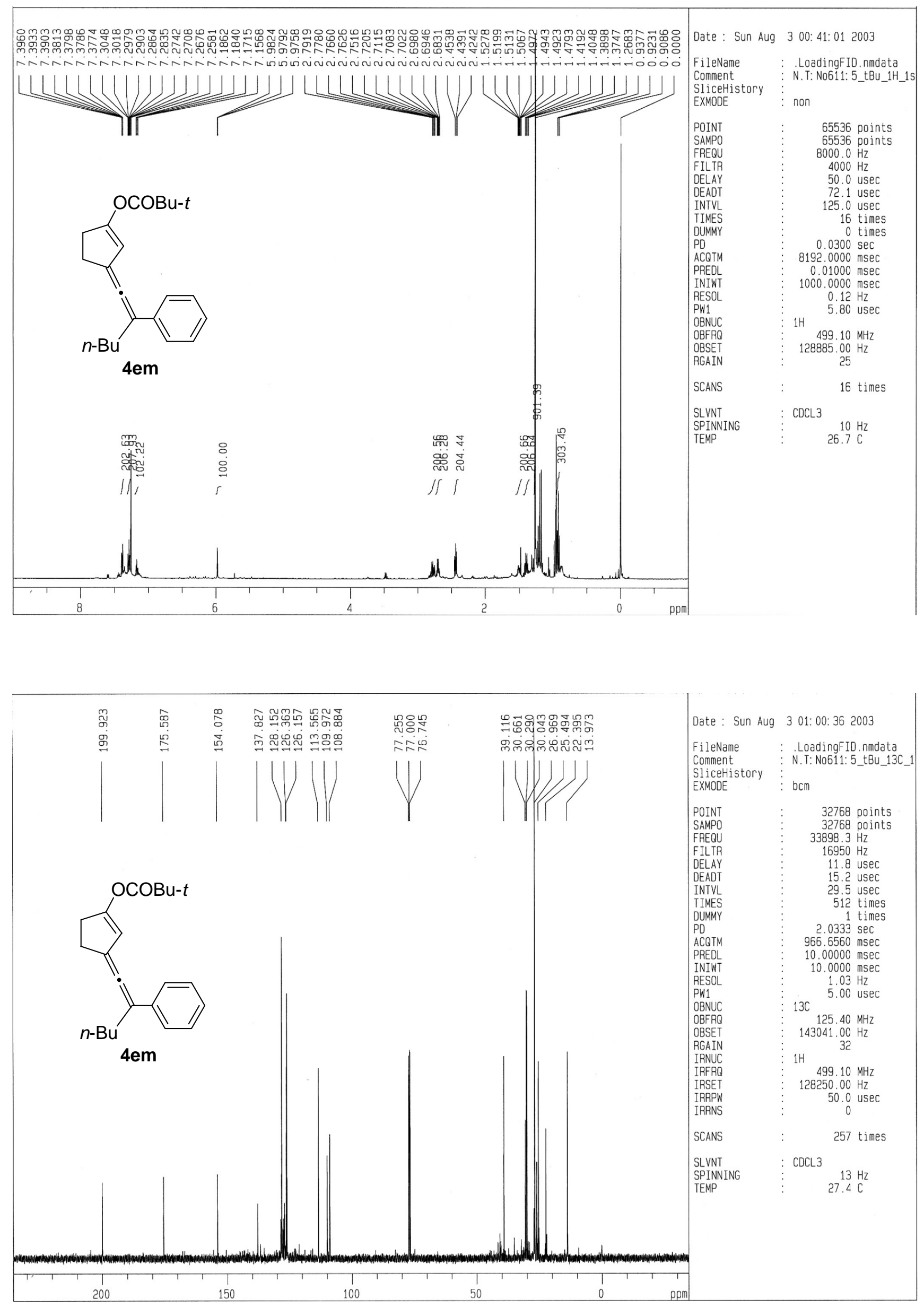

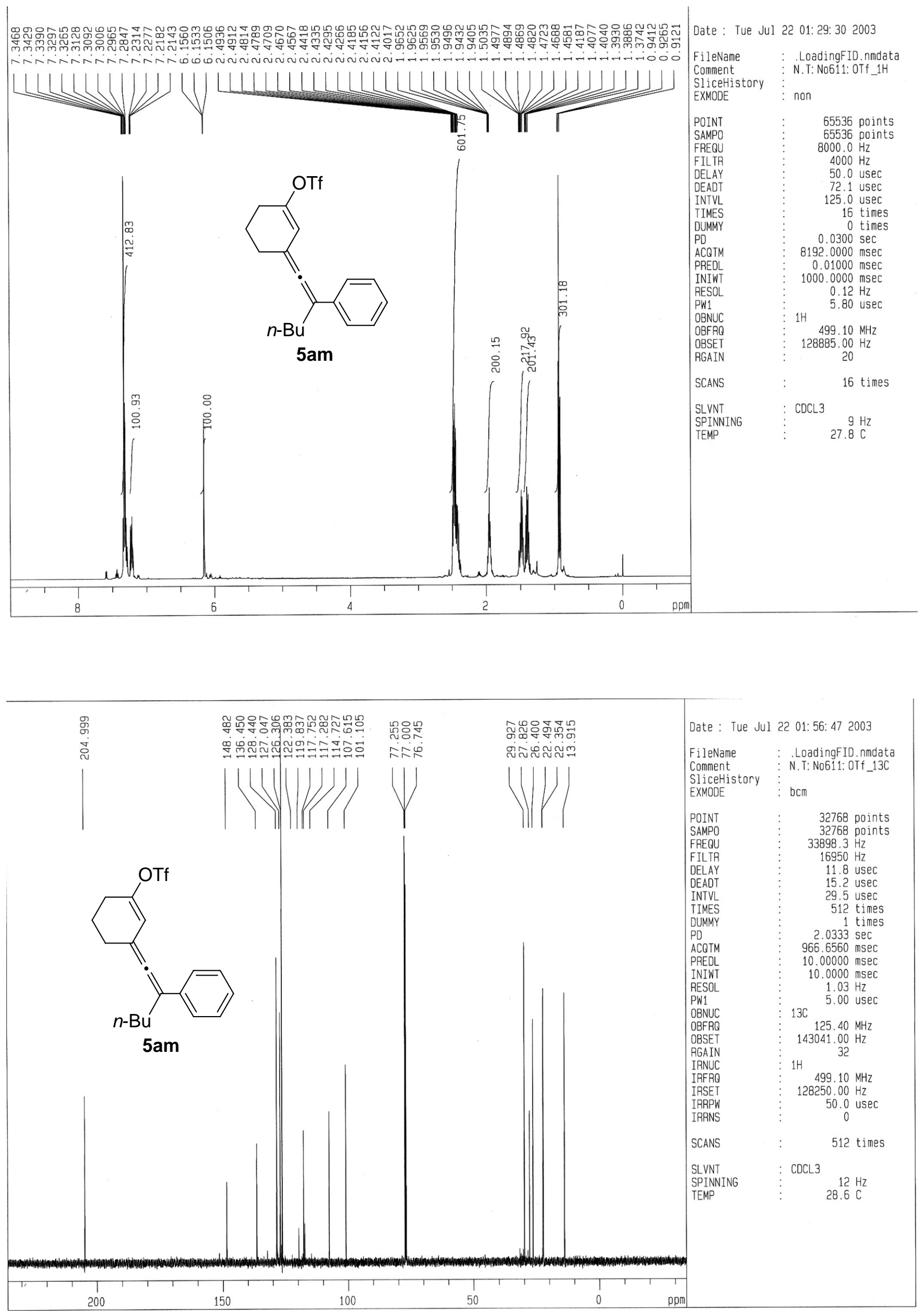